\title{
Article \\ The Amber Project: A Survey of Methods and Inks for the Reproduction of the Color of Translucent Objects
}

\author{
Abigail Trujillo-Vazquez *, Harrie Fuller, Susanne Klein and Carinna Parraman
}

check for updates

Citation: Trujillo-Vazquez, A.; Fuller, H.; Klein, S.; Parraman, C. The Amber Project: A Survey of Methods and Inks for the Reproduction of the Color of Translucent Objects. Appl. Sci. 2022, 12, 793. https://doi.org/ 10.3390/app12020793

Academic Editor: Esther Perales

Received: 1 November 2021 Accepted: 22 December 2021 Published: 13 January 2022

Publisher's Note: MDPI stays neutral with regard to jurisdictional claims in published maps and institutional affiliations.

Copyright: (C) 2022 by the authors. Licensee MDPI, Basel, Switzerland. This article is an open access article distributed under the terms and conditions of the Creative Commons Attribution (CC BY) license (https:// creativecommons.org/licenses/by/ $4.0 /)$.
Centre for Print Research, University of the West of England, Bristol BS16 1QY, UK; Harrie.Fuller@uwe.ac.uk (H.F.); Susanne.Klein@uwe.ac.uk (S.K.); Carinna.Parraman@uwe.ac.uk (C.P.)

* Correspondence: Abigail.TrujilloVazquez@uwe.ac.uk

Featured Application: Methods for the reproduction of the color of translucent objects by printing structural color are the first steps in the generation of a 3D effect in 2D and 2.5D prints. They will find applications not only in decorative printing but also in security printing since they are hard to duplicate by standard methods.

\begin{abstract}
Unlike regular pigments based on selective light absorption, the so-called "effect pigments" are based on the phenomena of structural color, or selective reflectance. Structural color has appealing aesthetic qualities, such as angle-dependent hue, and is able to produce lightfast colors. When used as a pigment, however, the gamut of the print is more limited, the color is difficult to measure, and therefore color management and preprint process become challenging. The aim of this paper is to compare the behavior of effect pigments in the processes of lithographic and screen printing with standard pigments used in so-called process inks, and to analyze their optical properties when used on their own or in combination with absorption pigments. An image of amber beads was printed as screen prints and lithographs. Three sets of inks were used: Set one: Standard process inks in the colors cyan, magenta, yellow and black (CMYK); set two: RGB inks formulated with Merck Spectraval ${ }^{\mathrm{TM}}$ pearlescent pigments which allow additive red, green, blue printing on a black substrate; and set three: golden inks formulated with pigments from the Merck Iriodin ${ }^{\mathrm{TM}}$ and Pyrisma ${ }^{\mathrm{TM}}$ effect pigment range. The image was printed on white and black paper. The optical appearance was assessed visually, and spectra and color coordinates were measured.
\end{abstract}

Keywords: CMYK; color coordinates; effect pigments; printing; RGB; spectral; structural color

\section{Introduction}

Color is an illusion [1], and its perception will change with lighting conditions, surface structure of the substrate, method, pigment choice, and the emotional state of the observer [2,3]. Generating color in a print-whether in a traditional 2D print or modern 3D print-that comes close to either the original coloration or the creator's idea has been recognized as difficult since the invention of print. Perceived color is hard to quantify and with the advent of new printing and coloration methods, it has become even more difficult [4]. Even today, soft and hard proofing, the quality control of color reproduction, are done by human observers [5].

At the beginning of the 21st century, Merck commercialized a new kind of pigments, the so-called effect pigments [6-8], which generate color by the interference of light. They are mainly marketed for cosmetic applications, car varnishing and packaging. We were interested in the characterization of the pigments when printed. The result of a discussion with Merck is the so-called 'amber project'. It is the attempt to reproduce the optical appearance of amber in print, ideally capturing color changes as a function of viewing angle and generating a 3D effect. As printing methods, we used the two primary commercial industrial printing processes: lithography and screen printing. Lithography is a planographic 
method of printing based on the antipathy of oil and water. The print matrix is either a grained flexible aluminum plate or porous limestone block. The industrial version of stone lithography is photo-offset lithography or short offset. In offset printing, the image is not directly transferred from the plate to the substrate but first onto the so-called blanket, a rubber sheet mounted on a cylinder. The rubber cylinder is flexible and allows to print on a variety of materials including wood, metal, fabric, leather and rough paper or cardboard. The image on the final substrate has the same orientation as on the plate since it has been transferred twice. The advantage of offset printing is that it produces a consistently high image quality, quick and easy production of the printing plate and low cost. Modern offset machines are fully automated and allow a fast turnover of print jobs with an hourly production of about 15,000 prints [9].

Screen printing was first mentioned about AD221 in China [10] and was used to print on fabric. The Japanese used paper of parchment stencils on screens made from human hair. It was introduced to Europe, but the technique only took off when photosensitive emulsions were developed in the early part of the 20th century [11]. Screen printing or serigraphy had a significant impact on the art world when in the 1960s Pop Art artists such as Peter Blake, Robert Rauschenberg and Andy Warhol used it to democratize art. Since then, it has found its application in many fields. In modern screen printing, the screen is a nylon or polyester mesh stretched over a frame. The first step is to coat the screen with a photosensitive emulsion. When the emulsion is dry, the coated screen is exposed to UV radiation through either stencils, transparent photographic images, or drawings on transparent film. After washing the non-exposed parts of the image away and drying the screen, the screen is positioned on the substrate and ink is forced through the screen with the help of a floodbar or squeegee. Since the screen is flexible and not much pressure is needed to apply the ink, the surface does not need to be flat and screen print can be applied to a large variety of substrates such as fabric, glass, metal, wood and paper. Industrially, three types of presses are common: flatbed, cylinder, and rotary. The cylinder press refers to the substrate being a cylinder or mounted on a cylinder. In a rotary press the screen is a cylinder.

The major difference between a lithograph and a screen print is the thickness of the ink layer. In a lithograph the ink layer has a thickness of 1-3 $\mu \mathrm{m}$ [12], whereas screen prints present a layer thickness of 15 to $30 \mu \mathrm{m}$ [13].

\section{Materials and Methods}

Three strings of amber beads and one large stone, all different colors and textures (Figure 1) were photographed under studio lighting, using an iPhone 12 pro, Apple ProRAW. The image in Figure 1 was printed as screen prints and lithographs with classic cyan, magenta, yellow and black inks (CMYK), Merck Spectraval ${ }^{\mathrm{TM}}$ pearlescent inks in red, green, and blue (RGB) and Merck Iriodin ${ }^{\mathrm{TM}}$, and Pyrisma ${ }^{\mathrm{TM}}$ effect pigments in Icy White, Platinum Gold, Solar Gold, Ambercup Orange (gold). White and black paper was used as substrates.

\subsection{Detail of the Two Different Printing Methods}

\subsubsection{Lithography}

We used aluminum plates which are coated with a layer of o-quinone diazides, diazo phenols and their derivatives [14]. Upon exposure to UV light, they become soluble in Ipagsa Developer HF-LD (5 to $20 \%$ disodium metasilicate in water). The image is transferred to the plate by exposing the plate through a halftone image on a transparent film, which makes the hydrophobic plate hydrophilic where the UV light is transmitted through the film. The surface is then etched using a solution of disodium metasilicate in water and varnished with Gum Arabic. As the surface of the printing plate is rolled with ink, the marks of the image on the plate, opaque areas on the halftone film, attract the oil-based ink and repel water; the blank etched areas (transparent areas on the halftone film) are hydrophilic, which attract water and repel ink. When printing, the plate is kept damp with water to maintain these conditions. We printed the image by employing direct 
lithography. Paper is placed onto the surface, and the plate is run through a press under pressure. The resulting image is a mirror image of the image on the plate.

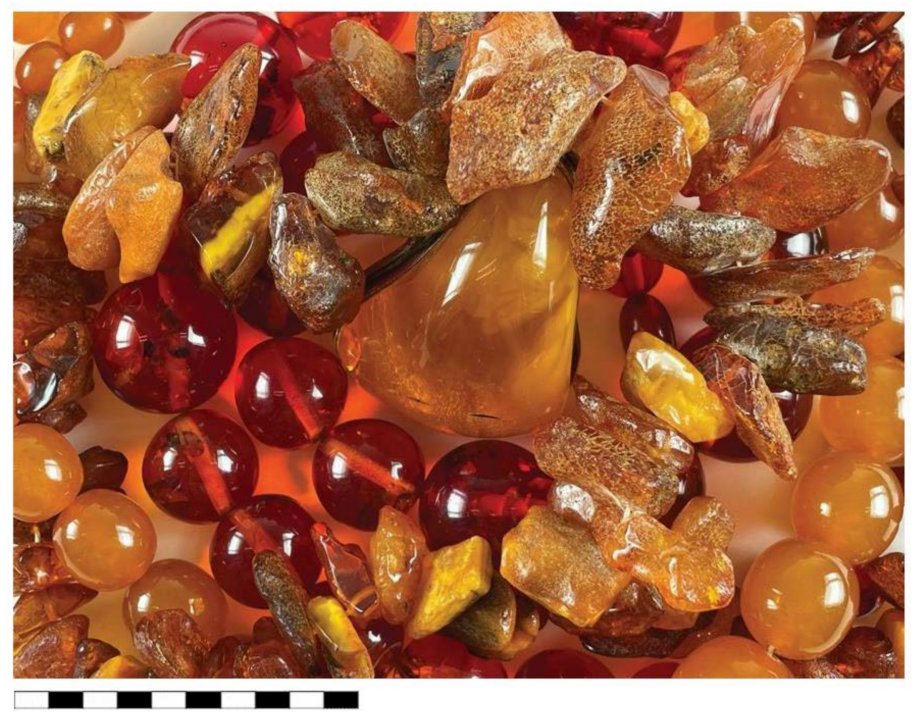

Figure 1. Digital image of various types and shapes of amber. Scale in $\mathrm{cm}$.

\subsubsection{Screen Printing}

The first step is to coat the screen with a photosensitive emulsion; we used Azacol Z1. When the emulsion is dry, the coated screen is exposed to UV radiation through either stencils, transparent photographic images, or drawings on transparent film. After washing the non-exposed parts of the image away and drying the screen, the screen is positioned on the substrate and ink is forced through the screen with the help of a floodbar or squeegee.

\subsection{Inks}

For the classic lithograph CMYK print, we used Primebio ${ }^{\mathrm{TM}}$ by van Son, a vegetable oil-based, high gloss, low tac offset ink. We followed the traditional layer sequence: Yellow was printed first, followed by magenta, cyan, and black. The RGB and gold inks were formulated in our research facilities. Spectraval ${ }^{\mathrm{TM}}$, Iriodin ${ }^{\mathrm{TM}}$ and Pyrisma ${ }^{\mathrm{TM}}$ effect pigments were provided by Merck KGaA. The pigments are titanium dioxide-coated mica plates with an average diameter ranging from 1 to 60 micrometers, see Table 1.

Table 1. Size range of Merck specialty pigments used in the amber project. The size range was provided by Merck.

\begin{tabular}{cc}
\hline Pigment Name & Size Range $(\mu \mathrm{m})$ \\
\hline Spectraval Red & $5-25$ \\
Spectraval Green & $5-25$ \\
Spectraval Blue & $1-20$ \\
Iriodin Icy White & $5-40$ \\
Iriodin Platinum Gold & $10-60$ \\
Iriodin Solar Gold & $10-60$ \\
Pyrisma Amber cup Orange & $5-35$ \\
\hline
\end{tabular}

The lithographic inks were made from linseed oil, effect pigment and talc (hydrated magnesium silicate). Talc was necessary to improve the rheology of the ink, make the ink short, increase drying and increase the light path in the ink layer via scattering which leads to a more saturated color. A typical recipe for a lithographic ink is in Table 2. 
Table 2. Typical recipe for lithographic inks with effect pigments as colorant.

\begin{tabular}{cc}
\hline Ingredient & Percentage by Weight \\
\hline Effect pigment & 30 \\
Talc & 20 \\
Linseed oil, 45 poise & 40 \\
Linseed oil & 10 \\
\hline
\end{tabular}

The effect pigment inks show liquid crystalline behavior and must be aligned during the printing process which is not straight forward in offset lithography. A better saturation was achieved when we printed direct "litho", the paper is in contact with the plate, which has only one ink split, compared to offset which has two splits.

For the screen-printing inks, Daler Rowney's System 3 was used as printing medium. For the CMYK prints, the medium was mixed with System 3 acrylic paint. Process cyan, process magenta, and process yellow were used as a $50 \%$ mix and process black was used at $30 \%$ by weight. The layer sequence was yellow, magenta, cyan, and black.

For the specialty inks, Daler Rowney's System 3 printing medium was used, mixing them with the powdered pigments. Two variations with Spectraval ${ }^{\mathrm{TM}}$ pigments were done: one with twice as much pigment (Table 3). We needed to vary the exact percentages depending on the pigments to achieve a balanced RGB color gamut. The reason for making up a second batch using half the amount of pigment is due to the thick layer of ink put down in screen print. When the three pigments are printed on top of each other on black paper, they appear to be white. If the ink is too pigmented, the color in the printed image becomes desaturated and can easily look 'over exposed'. For the Iriodin ${ }^{\mathrm{TM}}$ and Pyrisma ${ }^{\mathrm{TM}}$, we mixed them with the System 3 printing medium at $10 \%$ by weight $-40 \mathrm{~g}$ pigment, $400 \mathrm{~g}$ medium for all four colors.

Table 3. Content of Spectraval ${ }^{\mathrm{TM}}$ pigment and medium in screen printing inks.

\begin{tabular}{ccc}
\hline Color & Pigment & Medium \\
\hline Red 1 & $36 \mathrm{~g}$ & $400 \mathrm{~g}$ \\
Green 1 & $48 \mathrm{~g}$ & $400 \mathrm{~g}$ \\
Blue 1 & $40 \mathrm{~g}$ & $400 \mathrm{~g}$ \\
Red 2 & $18 \mathrm{~g}$ & $400 \mathrm{~g}$ \\
Green 2 & $24 \mathrm{~g}$ & $400 \mathrm{~g}$ \\
Blue 2 & $20 \mathrm{~g}$ & $400 \mathrm{~g}$ \\
\hline
\end{tabular}

\subsection{Paper}

To avoid misalignment of the effect pigments, we used 240 gsm (grams per square meter) Plike paper sold by G.F. Smith in white and black. The paper is highly calendared and has a very smooth, plastic like (hence the name) surface. A heavy paper was necessary when screen printing with a $55 \mathrm{H}$ mesh, a mesh count usually used for fabric. More ink is deposited on the substrate than with finer meshes commonly used for printing on paper. The high weight per area prevents the paper from wrinkling too much and enabled us to mantain the print registration.

\subsection{Halftoning}

The halftone dote sizedot was determined by the maximum pigment diameter, i.e., $60 \mu \mathrm{m}$. The mesh count was chosen based on the convention that the mesh opening has to be at least 3 times the pigment size. Iriodin ${ }^{\mathrm{TM}}$ Solar Gold pigments are the largest pigments used here and have plates of 10-60 $\mu \mathrm{m}$ in diameter [15] and of $0.5 \mu \mathrm{m}$ thickness [6]. Assuming a median of $35 \mu \mathrm{m}$ for the diameter, we decided to use a $55 \mathrm{H}$ mesh with a pore size of $105 \mu \mathrm{m}$.

Lines per inch or LPI is the measurement used to calculate the resolution of images printed in halftone; a smaller number gives a larger dot size. If the dots were too small, 
they would 'fall through' the holes in the $55 \mathrm{H}$ mesh. A standard procedure to calculate an adequate dot size is to consider: US mesh measurement/4.5 = LPI. Since 55 UK threads per $\mathrm{cm}=140$ USA threads per inch, the halftone recommended by this formula was 31 LPI [16]. We also needed to consider that for each dot to expose correctly without pinholes, they needed to be supported from three sides by the grid that the woven thread makes up. To ensure this, we used a slightly lower LPI than recommended by the formula. The last consideration was that a well-defined halftone pattern could only be achieved when the minimum dot size is larger than the maximum pigment diameter. Taking these three factors into account, we chose to use 25LPI.

The angular resolution of the human eye is about 1 arcminute, [17] which means the human observer can see the difference between two lines separated by $30 \mathrm{~cm}$ at a distance of $1 \mathrm{~km}$, equivalent to $0.09 \mathrm{LPI}$, or $0.12 \mathrm{~mm}$ at a viewing distance of $40 \mathrm{~cm}$, equivalent to about 210 LPI. A measurement of 25 LPI requires a viewing distance of a minimum $4.16 \mathrm{~m}$.

The viewing distance is coupled to the size of the image. We base the following calculations of the image size on the central visual field which is about $30^{\circ}$ [18]. The whole image can be seen clearly without eye movement within the central visual field. The dimensions of the print should be smaller than the diameter of the visual field at a distance of $4.16 \mathrm{~m}$ which is $2.23 \mathrm{~m}$. As the dimensions for our prints, we chose $600 \mathrm{~mm} \times 450 \mathrm{~mm}$, well within the above.

\subsection{Color Separations and Screen Angles}

To expose our lithograph plates and screen print, transparent films with imagery and halftone were created. We used Photoshop and the Raster Image Processor (RIP) software Film Master 10 to create the color separation and halftone with the appropriate screen angle to minimize moiré caused by the halftone patterns in the different layers. The color layers were printed as grayscale images on Astrajet inkjet film.

For standard CMYK prints the positive digital image was converted into a CMYK color profile using PhotoShop and then RIP software to separate the channels, apply screen angles and apply the halftone. Screens and plates were made from these films, and a full color image was generated by overprinting yellow with magenta, cyan and black on white paper. The same screens and plates were used for Iriodin ${ }^{\mathrm{TM}}$ and Pyrisma ${ }^{\mathrm{TM}}$ inks on white paper.

As the Spectraval ${ }^{\mathrm{TM}}$ pigments print the highlights on black paper, we needed to print negative images. The original image was separated into RGB in Photoshop using the channels function, and then inverted each layer. The three channels were then imported into the RIP software and halftone and screen angles were applied according to Table 4.

Table 4. Screen angles used.

\begin{tabular}{cc}
\hline Color Separation & Screen Angle \\
\hline Cyan positive & 105 \\
Magenta positive & 75 \\
Yellow positive & 0 \\
Black positive & 45 \\
Red negative & 15 \\
Green negative & 75 \\
Blue negative & 45 \\
Cyan negative & 105 \\
Magenta negative & 75 \\
Yellow negative & 0 \\
Black negative & 45 \\
\hline
\end{tabular}

\subsection{Printing Order}

We used the standard printing order of yellow, magenta, cyan and black to print the CMYK process inks. Since shadows are printed on white paper, the darker tones are printed last in order to achieve the needed contrast. 
Spectraval ${ }^{\mathrm{TM}}$ pigments were printed in the RGB order. This allows the pigments to align and mantains the tonality of the image. Theoretically, the printing order of the structural color inks should not affect the final color gamut since the particles are selectively reflecting, i.e., the rest of the spectrum is transmitted, but a reversal of the printing order to BGR shifted the overall color to red tones.

For the Iriodin ${ }^{\circledR}$ and Pyrisma ${ }^{\mathrm{TM}}$ specialty pigments, tests were made to find the correct printing order. According to the supplier (Merck) the darkest colors had to be printed on the darkest plate. Initially, following the principles of CMYK printing, the lighter tones were printed first and the darker ones last. We assessed the result of printing the darkest color on the darkest plate but switching the order of printing.

Printing light to dark, that is, the densest plate is printed last, led to a contrast loss. We inversed the printing order and the image quality improved but Iriodin ${ }^{\mathrm{TM}}$ Icy White and Platinum Gold did not show up on white paper. This led us to use them in a similar way to Spectraval ${ }^{\mathrm{TM}}$ based inks, printing the highlights onto black paper. New transparency films were made by separating the CMYK image in Photoshop using channels and then inverting each layer to create negative images. Screen angles and halftone were produced again with the RIP software. We achieved an image with contrast by printing a negative image dark to light on black Plike. However, the printed image had little tonality due to the narrow gamut of the speciality pigments.

\subsection{Measurements}

Transmittance spectra of the process inks cyan, magenta, and yellow were measured using a HP UV-Vis spectrometer. The inks are very absorbent. Instead of diluting the inks in linseed oil, which is yellow, we applied a thin layer on a microscope slide to avoid a color shift caused by the oil. Transmittance spectra for effect pigments are hard to interpret and therefore we measure reflectance spectra using a colorimeter X-Rite i1Profiler. The device uses LED Illumination (including UV), has an aperture of $4.5 \mathrm{~mm}\left(0.18^{\prime \prime}\right)$ diameter and an illumination spot size of $3 \mathrm{~mm}\left(0.12^{\prime \prime}\right)$. The color measurements were taken in the M1 mode for an observer at 2 degrees. The color viewing standard ISO 3664:2009 was used for this mode, as the light source contains UV content and complies with the spectral distribution specified by CIE illuminant D50. Measurements were saved as a "i1Profiler CGATS Custom" file type, which has a .txt extension, so the content can be easily read. The data files include a list of reflectance intensity values in intervals of $10 \mathrm{~nm}$ in the range of 380-730 nm, as well as color coordinates in CIE (1976) and XYZ color systems.

The CIELAB, or CIEL* $\mathrm{a}^{*} \mathrm{~b}^{*}$ color system represents quantitative relationships of colors on three axes: The $\mathrm{L}^{*}$ value indicates lightness, and $\mathrm{a}^{*}$ and $\mathrm{b}^{*}$ are chromaticity coordinates. On the color space diagram, $\mathrm{L}^{*}$ is represented on a vertical axis with values from 0 (black) to 100 (white). The $\mathrm{a}^{*}$ value indicates the red-green component of a color, where $+\mathrm{a}^{*}$ (positive) and $-\mathrm{a}^{*}$ (negative) indicate red and green values, respectively. The yellow and blue components are represented on the $b^{*}$ axis as $+b^{*}$ and $-b^{*}$ values, respectively. The center of the color space is achromatic. The distance from the central axis represents the chroma (C) or saturation of the color. The angle on the chromaticity axes represents the hue (h) [19].

\section{Results}

\subsection{Spectral Properties of the Inks}

Transmittance and reflectance spectra of the Cyan Magenta and Yellow inks were measured and an example is shown in Figures 2 and 3a. The reflectance spectra measured from the Spectraval ${ }^{\mathrm{TM}}$, Iriodin ${ }^{\mathrm{TM}}$ and Prysma ${ }^{\mathrm{TM}}$ is shown in Figure $3 \mathrm{~b}$.

Transmission and reflectance spectra may be useful as qualitative descriptions of the inks used in this work. For transmission, ink was rolled on glass slides and measured with an HP UV-vis spectrophotometer on transmission mode. Along with the reflectance curves, color coordinates were acquired. The average of three measurements with their standard deviation is shown in Table 5 and represented in a color space CIE (1976). The values of $b^{*}$ vs. $a^{*}$ from Table 5 are represented in the CIELab color space in Figure 4. 


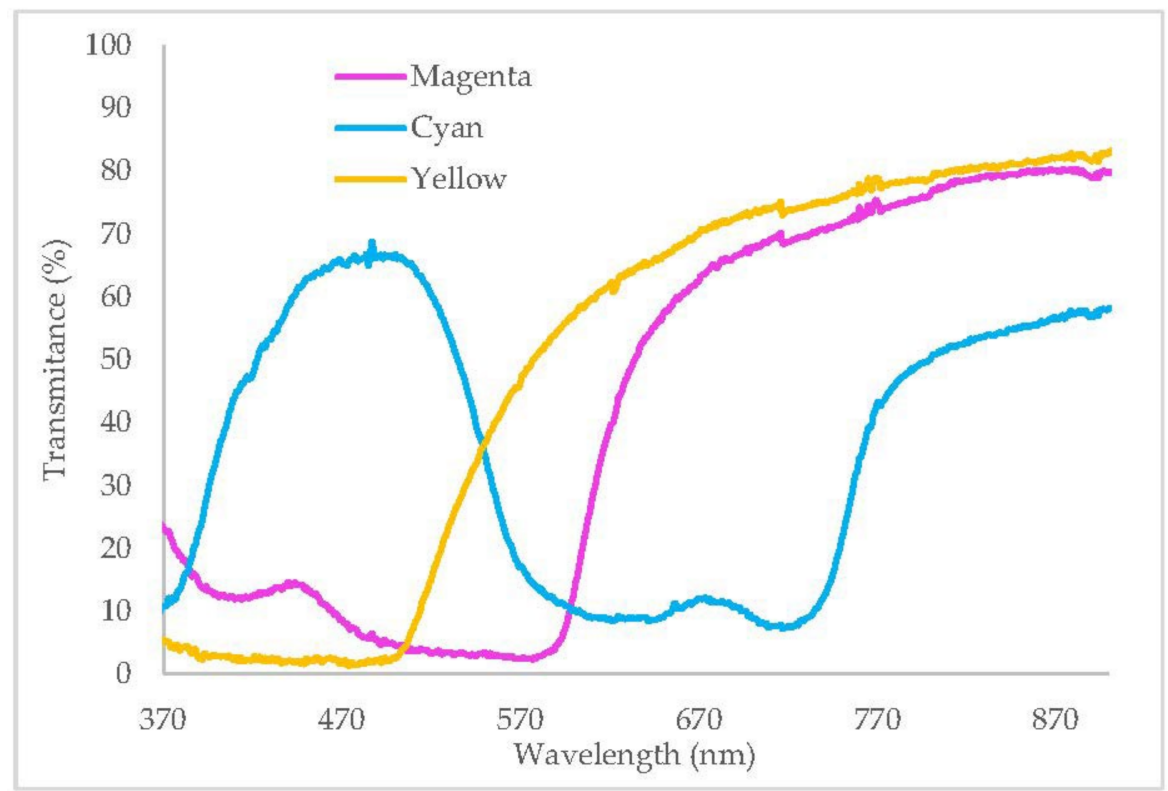

Figure 2. Transmittance spectra of lithography inks measured with a HP UV-vis spectrometer. The ink was rolled onto glass slides.

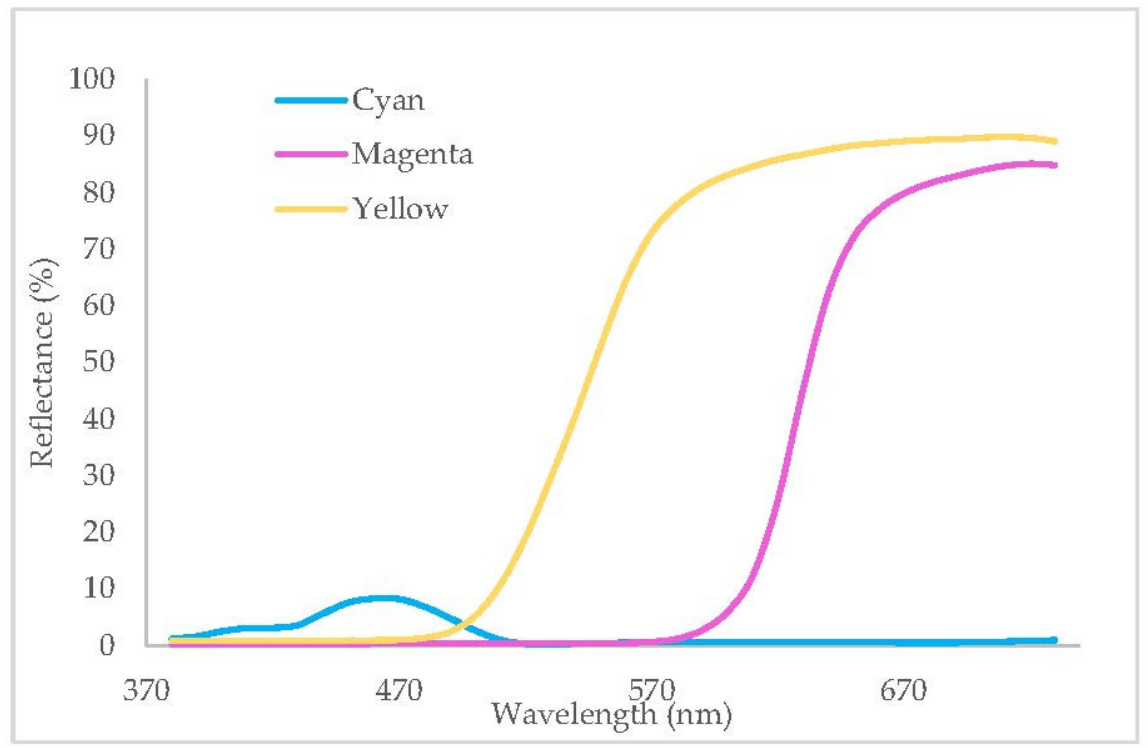

(a)

Figure 3. Cont. 


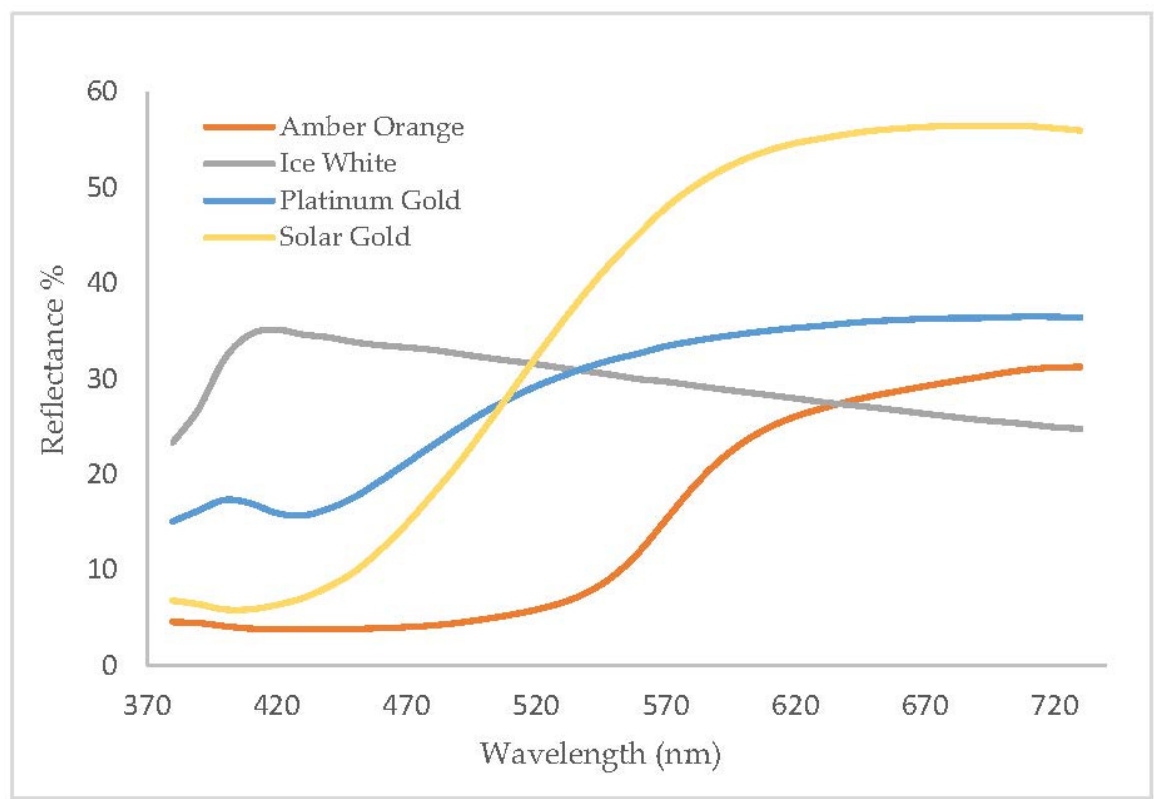

(b)

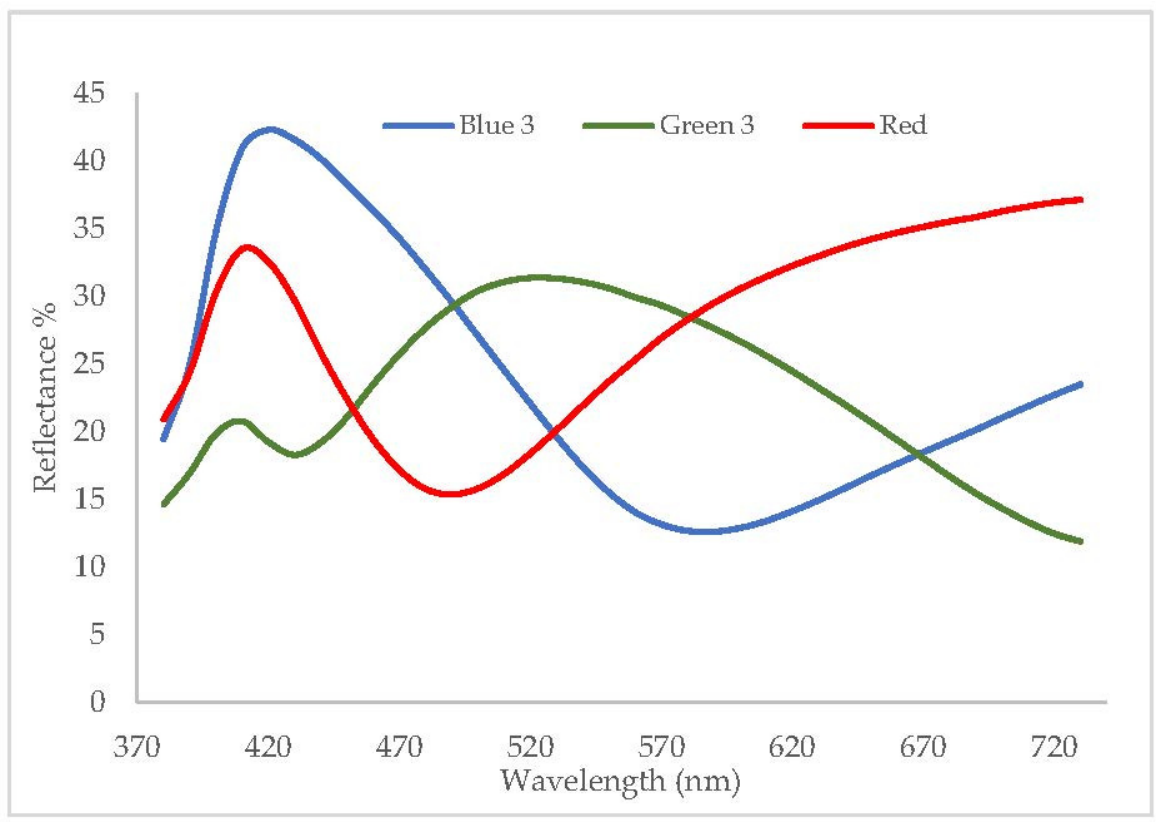

(c)

Figure 3. (a) Reflectance of Van Son Process Cyan, Magenta and Yellow inks on. (b) Reflectance of Spectraval $^{\mathrm{TM}}$ Iriodin $^{\mathrm{TM}}$ and Prysma ${ }^{\mathrm{TM}}$ inks (c) Reflectance of Spectraval ${ }^{\mathrm{TM}}$ inks. All measurements made with i1Pro colorimeter.

Table 5. Color measurements of inks on black Plike paper. The associated uncertainty of $L^{*}, a^{*}$ and $\mathrm{b}^{*} \mathrm{w}$ is the standard deviation of the measurements.

\begin{tabular}{ccccccc}
\hline Code & $\mathbf{L}^{*}$ & $\mathbf{\Delta} \mathbf{L}^{*}$ & $\mathbf{a}^{*}$ & $\mathbf{\Delta} \mathbf{a}^{*}$ & $\mathbf{b}^{*}$ & $\boldsymbol{\Delta} \mathbf{b}^{*}$ \\
\hline Amber Orange & 46.94 & 2.66 & 29.18 & 2.74 & 38.24 & 3.19 \\
Blue 3 & 52.72 & 6.31 & 2.39 & 0.09 & -30.39 & 2.02 \\
Green 3 & 59.76 & 4.63 & -10.96 & 0.88 & 8.81 & 0.85 \\
Icy white & 67.29 & 5.38 & -2.02 & 0.12 & -4.44 & 0.66 \\
Platinum Gold & 62.99 & 0.43 & 0.44 & 0.07 & 20.34 & 0.56 \\
Red & 58.82 & 1.61 & 14.57 & 0.07 & 6.23 & 0.53 \\
Solar Gold & 68.84 & 3.44 & 6.15 & 0.65 & 47.01 & 4.50 \\
\hline
\end{tabular}




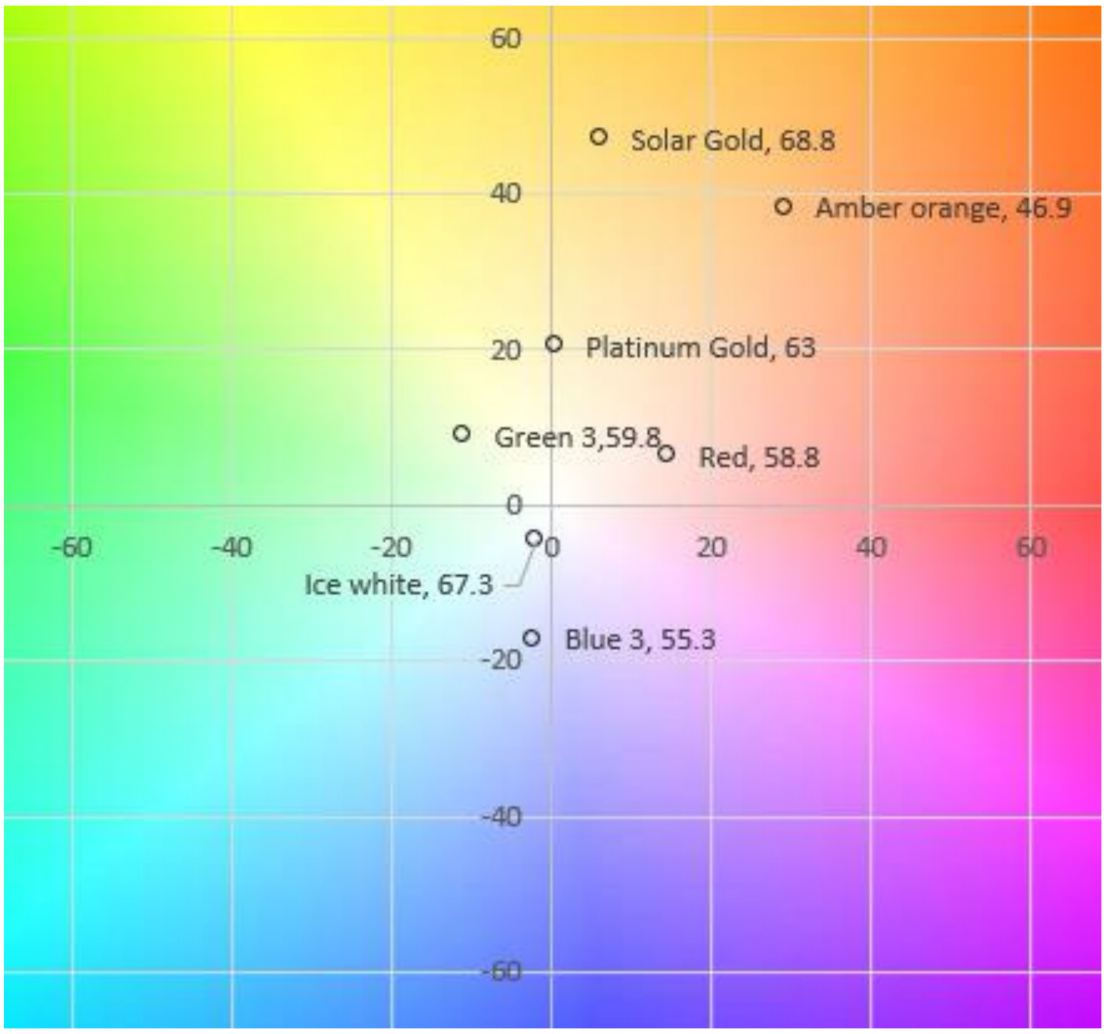

Figure 4. $a^{*}, b^{*}$ coordinates of the inks from Table 6 in the color space CIEL*a*b. The number in each label is the mean luminosity.

Table 6. Printed outcomes. S = Solar Gold. Symbol ' indicates the number of passes. I \& P means Iriodin $^{\mathrm{TM}}$ and Pyrisma ${ }^{\mathrm{TM}}$ specialty pigments.

\begin{tabular}{|c|c|c|c|c|c|}
\hline Code & Method & Paper ${ }^{1}$ & Film & Inks & $\begin{array}{l}\text { Image } \\
\text { Number }\end{array}$ \\
\hline S 5 & Screen Printing & Black & Negative & RGB 1 & 1 \\
\hline DL 15 & Direct Litho & White & Positive & S S on Y plate $+\mathrm{M}$ & 2 \\
\hline OL 1 & Offset Litho & White & Positive & YMCK & 3 \\
\hline DL 2 & Direct Litho & White & Positive & YMCK+ S & 4 \\
\hline DL 14 & Direct Litho & White & Positive & $S$ on $Y$ plate & 5 \\
\hline OL 13 & Offset Litho & Black & Positive & $\mathrm{I} \& \mathrm{P}, 1$ to $\mathrm{d}$ & 6 \\
\hline OL 9 & Offset Litho & Black & Negative & $\mathrm{R}^{\prime \prime} \mathrm{G}^{\prime \prime \prime} \mathrm{B}^{\prime \prime}$ & 7 \\
\hline DL 7 & Direct Litho & Black & Negative & BGR & 8 \\
\hline DL 4 & Direct Litho & Black & Negative & RGB & 9 \\
\hline OL 6 & Offset Litho & Black & Negative & $\mathrm{R}^{\prime \prime} \mathrm{G}^{\prime \prime \prime} \mathrm{B}^{\prime \prime \prime}$ & 10 \\
\hline OL 3 & Offset Litho & Black & Negative & $\mathrm{R}^{\prime \prime} \mathrm{G}^{\prime \prime \prime} \mathrm{B}^{\prime \prime \prime \prime}$ & 11 \\
\hline S 16 & Screen Printing & White & Positive & CMYK & 12 \\
\hline S 8 & Screen Printing & Black & Negative & RGB 2 & 13 \\
\hline S 12 & Screen Printing & White & Positive & $I \& P, 1$ to $d$ & 14 \\
\hline S 11 & Screen Printing & Black & Positive & $\mathrm{I} \& \mathrm{P}, \mathrm{d}$ to $\mathrm{l}$ & 15 \\
\hline S 10 & Screen Printing & Black & Negative & RGB 3 & \\
\hline
\end{tabular}

${ }^{1}$ All paper is G.F. Smith ${ }^{\circledR}$ Plike.

\subsection{Prints}

The methods used for reproducing the original image of amber were the following:

- Offset lithography (OL)

- Direct lithography (DL)

- $\quad$ Screen printing $(S)$

Table 6 summarizes the outcomes and their variations. 
The prints listed in Table 6 were used for measurements. A total of nine spots were chosen across the image and three measurements by spot were taken with an i1Pro colorimeter. The spots were areas of about $2 \mathrm{~cm}$ in diameter, therefore, the actual measuring area of the device ( $4 \mathrm{~mm}$ diameter) was in a random location within that area. The results reported here as CIEL* $a^{*} b^{*}$ color values are the average of the values measured in those three points, except in the cases where the signal was too weak to be considered reliable.

The measuring spots are shown in Figure 5 and are labeled as the following colors: Background 1, Background 2, Amber 1, Amber 2, Amber 3, Amber 4, Red 1, Red 2, and Red 3. To keep the spots' location constant across prints, a transparent sheet with 9 holes was placed and aligned on top of each print, and the measurements were taken through the holes (Figure 3). Tables 7-9, show the average measurements of $L^{*} a^{*} b^{*}$ values the spots labeled as Amber 3, Red 2, and Background 2. The uncertainty is the standard deviation of the measurements. For those cases where only one measurements was taken or the values of two measurements were identical no uncertainty is reported.

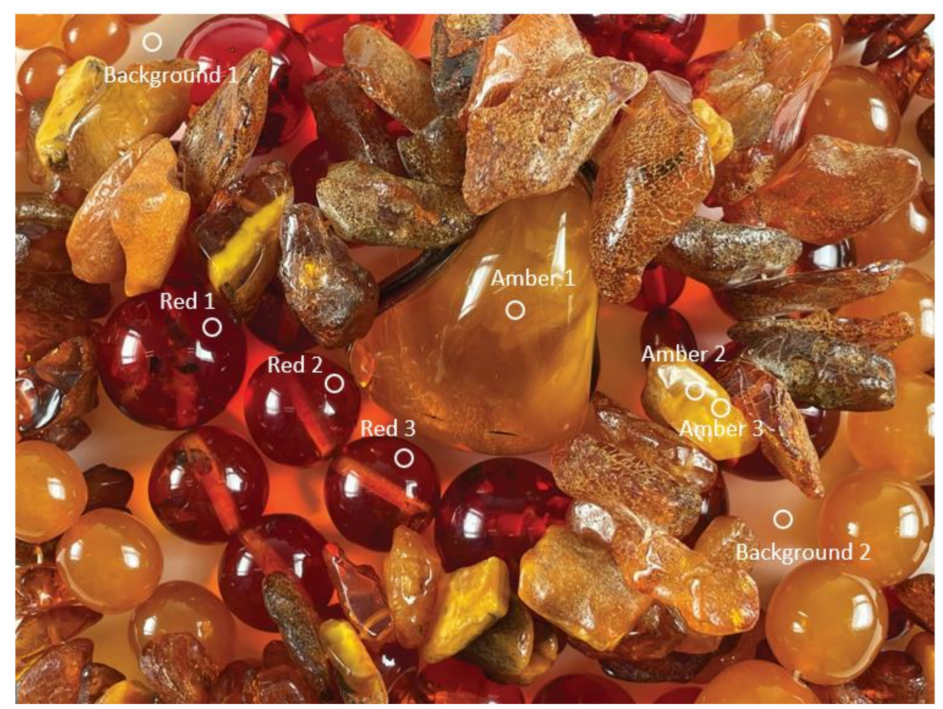

Figure 5. Original digital image of amber beads with spots where the measurements were taken.

Table 7. Average color measurements of Amber 3 sorted from largest to smallest $\mathrm{L}^{*}$.

\begin{tabular}{ccccccc}
\hline Code & $\mathbf{L}^{*}$ & $\boldsymbol{\Delta} \mathbf{L}$ & $\mathbf{a}^{*}$ & $\boldsymbol{\Delta} \mathbf{a}^{*}$ & $\mathbf{b}^{*}$ & $\Delta \mathbf{b}^{*}$ \\
\hline DL 14 & 88.80 & 0.66 & 2.45 & 0.27 & 20.28 & 0.67 \\
DL 15 & 82.43 & 0.67 & 14.67 & 0.67 & 11.55 & 0.67 \\
DL 2 & 78.41 & 1.74 & 11.17 & 2.09 & 26.20 & 3.09 \\
OL 1 & 77.99 & 1.63 & 9.22 & 2.49 & 52.08 & 2.54 \\
S 16 & 69.45 & 4.71 & 25.67 & 8.51 & 59.46 & 1.72 \\
S 5 & 63.29 & 0.43 & -0.90 & 0.24 & 5.99 & 0.27 \\
S 8 & 60.37 & 0.94 & 3.82 & 0.12 & 32.33 & 0.86 \\
S 12 & 56.87 & 0.45 & 22.06 & 1.90 & 35.56 & 1.39 \\
S 10 & 47.39 & 1.02 & 0.92 & 0.13 & 5.49 & 0.08 \\
S 11 & 45.02 & 1.68 & 13.28 & 1.64 & 31.95 & 0.35 \\
DL 4 & 40.45 & 0.76 & 0.41 & 0.31 & 2.10 & 0.14 \\
DL 7 & 38.58 & 0.66 & 8.48 & 0.59 & 0.12 & 0.06 \\
OL 9 & 29.84 & 0.56 & 0.36 & 0.12 & -0.23 & 0.20 \\
OL 6 & 29.27 & 0.98 & 1.17 & 0.50 & -0.75 & 0.74 \\
OL 13 & 26.93 & 0.17 & 2.47 & 0.10 & 2.97 & 0.33 \\
OL 3 & 26.59 & 0.00 & 0.26 & 0.00 & -0.51 & 0.00 \\
\hline
\end{tabular}


Table 8. Average color measurements of Red 2 sorted from largest to smallest L*.

\begin{tabular}{ccccccc}
\hline Code & $\mathbf{L}^{*}$ & $\mathbf{\Delta} \mathbf{L}$ & $\mathbf{a}^{*}$ & $\mathbf{\Delta} \mathbf{a}^{*}$ & $\mathbf{b}^{*}$ & $\boldsymbol{\Delta} \mathbf{b}^{*}$ \\
\hline DL 14 & 90.03 & 0.70 & 2.14 & 0.29 & 19.34 & 1.31 \\
DL 15 & 80.46 & 1.47 & 21.78 & 2.11 & 4.93 & 0.49 \\
S 12 & 63.11 & - & 9.20 & - & 28.52 & - \\
DL 2 & 60.90 & 0.01 & 1.85 & 0.20 & 24.80 & 1.04 \\
S 11 & 60.23 & - & 4.44 & - & 26.35 & - \\
S 8 & 46.39 & 0.00 & 8.26 & 0.23 & 32.64 & 0.12 \\
S 5 & 37.92 & 0.78 & 7.48 & 0.07 & -0.21 & 0.15 \\
S 16 & 34.73 & 1.84 & 32.25 & 3.61 & 14.21 & 0.83 \\
OL 1 & 33.49 & 1.25 & 41.61 & 0.39 & 22.16 & 1.93 \\
DL 7 & 32.31 & 0.08 & 4.69 & 0.04 & -1.02 & 0.00 \\
DL 4 & 30.90 & - & 2.43 & - & -0.91 & - \\
S 10 & 30.10 & 0.39 & 5.56 & 0.15 & -0.69 & 0.25 \\
OL 13 & 28.86 & 0.33 & 2.49 & 0.02 & 4.45 & 0.04 \\
OL 6 & 25.67 & - & 1.07 & - & -1.47 & - \\
OL 9 & 24.81 & 0.29 & 0.29 & 0.14 & -1.29 & 0.16 \\
OL 3 & 24.68 & - & 0.41 & - & -1.08 & - \\
\hline
\end{tabular}

Table 9. Average color measurements of Background 2 sorted from largest to smallest L*.

\begin{tabular}{ccccccc}
\hline Code & $\mathbf{L}^{*}$ & $\boldsymbol{\Delta} \mathbf{L}$ & $\mathbf{a}^{*}$ & $\boldsymbol{\Delta} \mathbf{a}^{*}$ & $\mathbf{b}^{*}$ & $\boldsymbol{\Delta} \mathbf{b}^{*}$ \\
\hline DL 14 & 88.42 & 0.35 & 2.73 & 0.13 & 21.28 & 0.46 \\
DL 15 & 79.59 & 0.38 & 19.72 & 0.58 & 8.71 & 0.45 \\
OL 1 & 72.28 & 5.85 & 7.90 & 0.82 & 12.61 & 2.28 \\
DL 2 & 69.99 & 0.10 & 9.87 & 0.98 & 23.16 & 0.16 \\
S 16 & 63.13 & 0.37 & 30.79 & 0.02 & 46.66 & 2.13 \\
S 5 & 60.12 & 0.79 & 0.31 & 0.51 & 3.05 & 0.12 \\
S 12 & 59.82 & 0.69 & 15.04 & 0.14 & 30.35 & 1.03 \\
S 8 & 59.21 & 1.16 & 4.10 & 0.27 & 26.84 & 1.44 \\
S 11 & 51.40 & 0.14 & 7.34 & 0.33 & 26.66 & 0.29 \\
S 10 & 43.86 & 0.46 & 1.48 & 0.17 & 1.01 & 0.29 \\
DL 7 & 43.59 & 1.29 & 6.36 & 1.12 & -1.09 & 0.35 \\
DL 4 & 37.97 & 1.94 & 0.59 & 0.35 & -0.74 & 0.45 \\
OL 6 & 32.64 & 5.43 & 5.12 & 2.87 & -0.59 & 0.25 \\
OL 9 & 28.92 & 0.68 & 0.20 & 0.06 & -2.49 & 0.37 \\
OL 3 & 26.35 & 0.00 & 0.02 & 0.00 & -2.36 & 0.00 \\
OL 13 & 25.86 & 0.25 & 1.73 & 0.20 & 2.06 & 0.23 \\
\hline
\end{tabular}

The values of $b^{*}$ vs. $a^{*}$ from the Tables 7-9 are represented in a section of the CIEL ${ }^{*} b^{*}$ color space in Figure 6. The values of Chroma (C) and hue (h) were calculated as [17].

$$
\mathrm{C}=\sqrt{\mathrm{a} *^{2}+\mathrm{b} *^{2}} \text { and } \mathrm{h}=\operatorname{atan} \frac{\mathrm{b} *}{\mathrm{c} *} .
$$




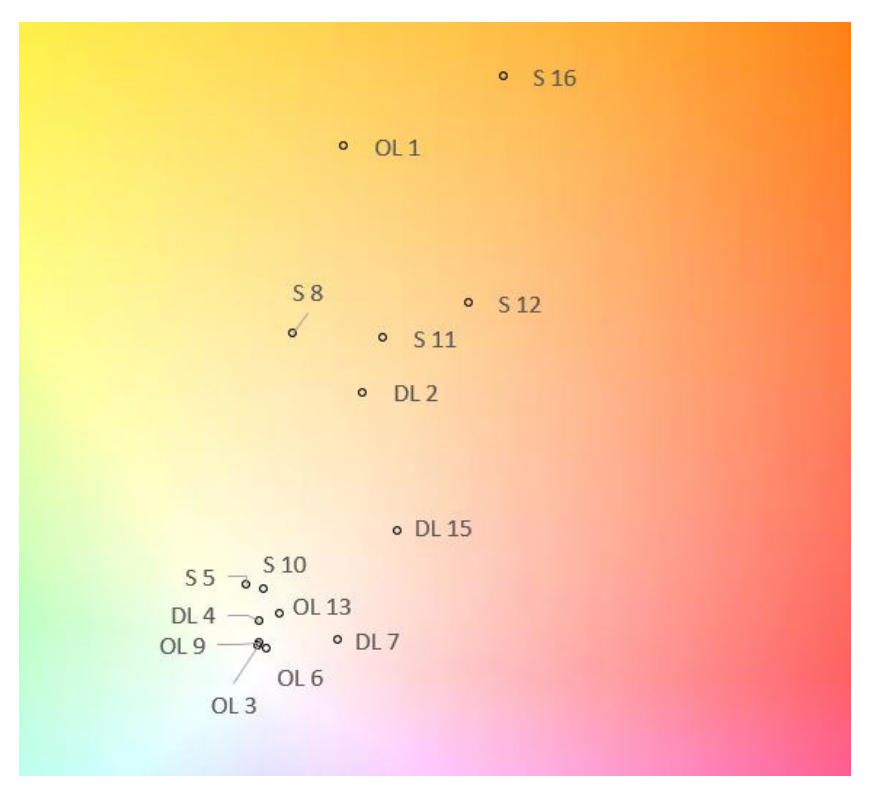

(a)

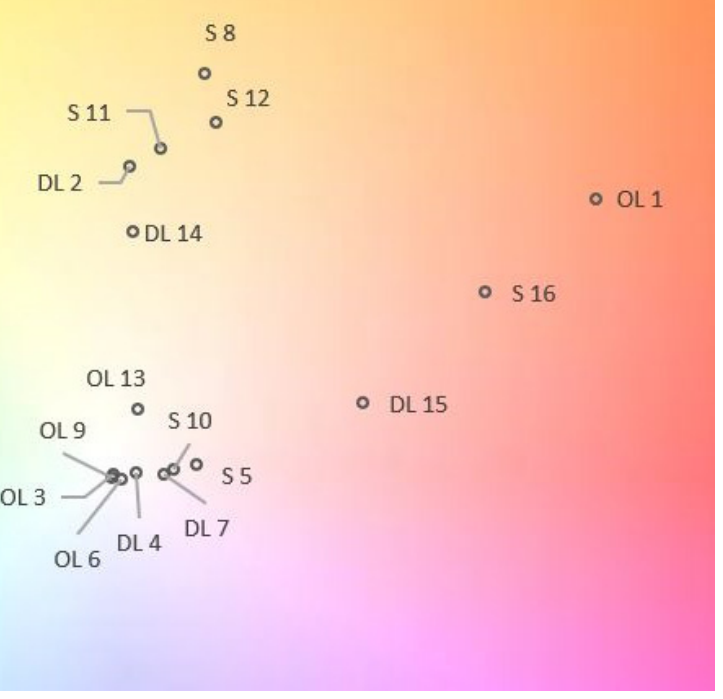

(b)

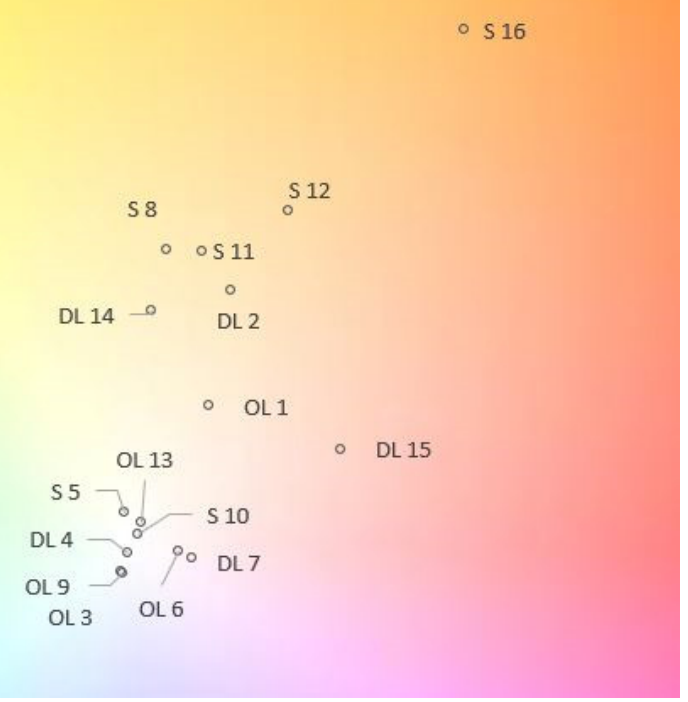

(c)

Figure 6. Representation of $\mathrm{a}^{*}$ and $\mathrm{b}^{*}$ coordinates in the color space CIEL ${ }^{*} \mathrm{a}^{*} \mathrm{~b}$ for a color spot in different prints. Measuring spots: (a) amber 2; (b) red 3; (c) background 2.

The average values of luminosity (L), chroma (C) and hue (h) and their uncertainties as standard deviation were calculated for all the amber, red and background spots. The results are shown in Tables 10-12. Information on the color of the paper, whether the film is positive or negative and an identifier of the ink is added to the table for an easier interpretation of the results. 
Table 10. Average luminosity, chroma and hue of all amber spots. Ordered according to the higher luminosity value.

\begin{tabular}{cccccccc}
\hline Code & $\mathbf{L}$ & $\mathbf{\Delta}$ & $\mathbf{C}$ & $\mathbf{\Delta C}$ & $\mathbf{h}$ & $\boldsymbol{\Delta} \mathbf{h}$ & Paper/Film/Ink \\
\hline DL 14 & 88.28 & 1.18 & 21.54 & 1.81 & 1.44 & 0.02 & White Positive S on Y plate \\
DL 15 & 80.28 & 0.76 & 21.00 & 0.45 & 0.45 & 0.09 & White Positive S on Y plate \\
DL 2 & 73.65 & 7.16 & 30.39 & 1.75 & 1.20 & 0.07 & White Positive YMCK+ S \\
S 16 & 69.45 & - & 64.76 & - & 1.16 & - & White Positive CMYK \\
OL 1 & 66.58 & 10.09 & 50.39 & 4.06 & 1.21 & 0.17 & White Positive YMCK \\
S 5 & 60.08 & 3.53 & 4.94 & 1.24 & -0.10 & 1.56 & Black Negative RGB 1 \\
S 8 & 57.73 & 2.41 & 33.48 & 0.79 & 1.44 & 0.01 & Black Negative RGB 2 \\
S 12 & 57.48 & 1.15 & 29.80 & 19.96 & 1.06 & 0.07 & White Positive I \& P, dark to light \\
S 11 & 49.53 & 4.35 & 31.37 & 3.20 & 1.24 & 0.06 & Black Positive I \& P, dark to light \\
S 10 & 45.70 & 3.75 & 4.94 & 1.38 & 0.30 & 1.57 & Black Negative RGB 3 \\
DL 4 & 39.27 & 1.06 & 1.70 & 0.64 & 0.99 & 0.57 & Black Negative S on Y plate \\
DL 7 & 36.70 & 3.91 & 6.17 & 2.18 & -0.04 & 0.04 & Black Negative BGR \\
OL 9 & 28.84 & 0.93 & 1.23 & 0.67 & -1.03 & 0.31 & Black Negative R $\mathrm{R}^{\prime \prime} \mathrm{G}^{\prime \prime \prime} \mathrm{B}^{\prime \prime}$ \\
OL 6 & 28.53 & 0.93 & 1.64 & 0.32 & -0.75 & 0.21 & Black Negative $\mathrm{R}^{\prime \prime} \mathrm{G}^{\prime \prime \prime} \mathrm{B}^{\prime \prime \prime}$ \\
OL 13 & 26.74 & 0.32 & 3.41 & 0.40 & 0.92 & 0.04 & Black Positive I \& P, light to dark \\
OL 3 & 26.30 & 0.34 & 1.14 & 0.48 & -1.27 & 0.17 & Black Negative $R^{\prime \prime} \mathrm{G}^{\prime \prime \prime} \mathrm{B}^{\prime \prime \prime \prime \prime}$ \\
\hline
\end{tabular}

Table 11. Average luminosity, chroma and hue of all red spots. Ordered according to the higher luminosity value.

\begin{tabular}{|c|c|c|c|c|c|c|c|}
\hline Code & $\mathbf{L}$ & $\Delta \mathrm{L}$ & $\mathrm{C}$ & $\Delta \mathrm{C}$ & $\mathbf{h}$ & $\Delta \mathrm{h}$ & Paper/Film/Ink \\
\hline DL 14 & 89.73 & 0.58 & 19.76 & 1.15 & 1.46 & 0.00 & White Positive S on Y plate \\
\hline DL 15 & 79.60 & 1.02 & 23.53 & 1.04 & 0.20 & 0.04 & White Positive S on Y plate \\
\hline S 12 & 64.33 & 1.73 & 30.15 & 0.26 & 1.29 & 0.04 & White Positive I \& $\mathrm{P}$, dark to light \\
\hline DL 2 & 61.62 & 0.67 & 25.23 & 1.65 & 1.44 & 0.14 & White Positive CMYK + S \\
\hline S 11 & 60.73 & 0.44 & 24.85 & 1.62 & 1.41 & 0.01 & White Positive YMCK \\
\hline S 8 & 46.39 & 0.58 & 29.38 & 6.44 & 1.30 & 0.04 & Black Negative RGB 2 \\
\hline S 5 & 37.25 & 1.66 & 7.33 & 0.51 & -0.05 & 0.09 & Black Negative RGB 1 \\
\hline S 16 & 34.73 & & 35.24 & & 0.41 & & White Positive CMYK \\
\hline OL 1 & 31.85 & 0.88 & 28.58 & 21.93 & 0.30 & 0.44 & White Positive YMCK \\
\hline DL 7 & 32.00 & 0.27 & 4.50 & 0.26 & -0.21 & 0.01 & Black negative BGR \\
\hline DL 4 & 29.51 & 1.31 & 2.33 & 1.63 & -0.40 & 0.05 & Black Negative S on Y plate \\
\hline S 10 & 29.41 & 1.03 & 5.40 & 0.22 & -0.12 & 0.07 & Black Negative RGB 3 \\
\hline OL 13 & 28.12 & 0.80 & 4.25 & 0.87 & 1.04 & 0.02 & Black Positive I \& P, light to dark \\
\hline OL 6 & 26.18 & 0.44 & 1.86 & 0.16 & -0.77 & 0.29 & Black Negative $\mathrm{R}^{\prime \prime} \mathrm{G}^{\prime \prime \prime} \mathrm{B}^{\prime \prime \prime}$ \\
\hline OL 9 & 25.13 & 0.33 & 1.38 & 0.06 & -1.25 & 0.14 & Black negative $\mathrm{R}^{\prime \prime} \mathrm{G}^{\prime \prime \prime} \mathrm{B}^{\prime \prime}$ \\
\hline OL 3 & 24.61 & 0.38 & 1.16 & 0.07 & -1.22 & 0.20 & Black Negative $\mathrm{R}^{\prime \prime} \mathrm{G}^{\prime \prime \prime} \mathrm{B}^{\prime \prime \prime \prime}$ \\
\hline
\end{tabular}


Table 12. Average luminosity, chroma and hue of all background spots. Ordered according to the higher luminosity value.

\begin{tabular}{|c|c|c|c|c|c|c|c|}
\hline Code & $\mathbf{L}$ & $\Delta \mathbf{L}$ & $\mathrm{C}$ & $\Delta \mathrm{C}$ & $\mathbf{h}$ & $\Delta \mathbf{h}$ & Paper/Film/Ink \\
\hline DL 14 & 90.90 & 3.50 & 18.79 & 3.77 & 1.49 & 0.07 & White Positive S on Y plate \\
\hline DL 15 & 80.21 & 0.88 & 21.39 & 0.24 & 0.37 & 0.06 & White Positive S on Y plate \\
\hline OL 1 & 69.34 & 4.16 & 22.36 & 10.58 & 0.98 & 0.05 & White Positive YMCK \\
\hline DL 2 & 74.78 & 6.77 & 20.59 & 6.49 & 1.10 & 0.09 & White Positive CMYK + S \\
\hline S 16 & 63.13 & & 55.90 & & 0.99 & 0.00 & White Positive YMCK \\
\hline S 5 & 60.79 & 0.95 & 1.65 & 2.01 & 0.93 & 0.76 & Black Negative RGB 1 \\
\hline S 12 & 59.82 & 0.00 & 33.87 & 0.00 & 1.11 & 0.00 & White Positive I \& $\mathrm{P}$, dark to light \\
\hline S 8 & 61.60 & 3.37 & 21.59 & 7.87 & 1.40 & 0.03 & Black Negative RGB 2 \\
\hline S 11 & 45.05 & 8.98 & 25.87 & 2.51 & 1.24 & 0.08 & White Positive YMCK \\
\hline S 10 & 44.64 & 1.10 & 1.68 & 0.16 & -0.42 & 1.45 & Black Negative RGB 3 \\
\hline DL 7 & 42.72 & 1.23 & 5.92 & 0.76 & -0.20 & 0.04 & Black negative BGR \\
\hline DL 4 & 38.26 & 0.40 & 1.46 & 0.73 & -0.63 & 0.38 & Black Negative $S$ on Y plate \\
\hline OL 9 & 32.03 & 0.86 & 3.73 & 2.02 & 0.46 & 0.81 & Black negative ${ }^{\prime \prime} \mathrm{G}^{\prime \prime \prime} \mathrm{B}^{\prime \prime}$ \\
\hline OL 6 & 29.15 & 0.32 & 2.83 & 0.47 & -1.34 & 0.21 & Black Negative $R^{\prime \prime} G^{\prime \prime \prime} B^{\prime \prime \prime}$ \\
\hline OL 3 & 27.67 & 1.86 & 2.04 & 0.45 & -0.31 & 1.76 & Black Negative $\mathrm{R}^{\prime \prime} \mathrm{G}^{\prime \prime \prime} \mathrm{B}^{\prime \prime \prime \prime}$ \\
\hline OL 13 & 25.35 & 0.72 & 1.86 & 1.17 & 0.77 & 0.14 & Black Positive I \& P, light to dark \\
\hline
\end{tabular}

The values of averaged luminosity, chroma and hue are plotted in Figures 7-9, respectively. The relation chroma vs. luminosity is shown in Figure 10 for the averaged of amber spots as an illustrative example.

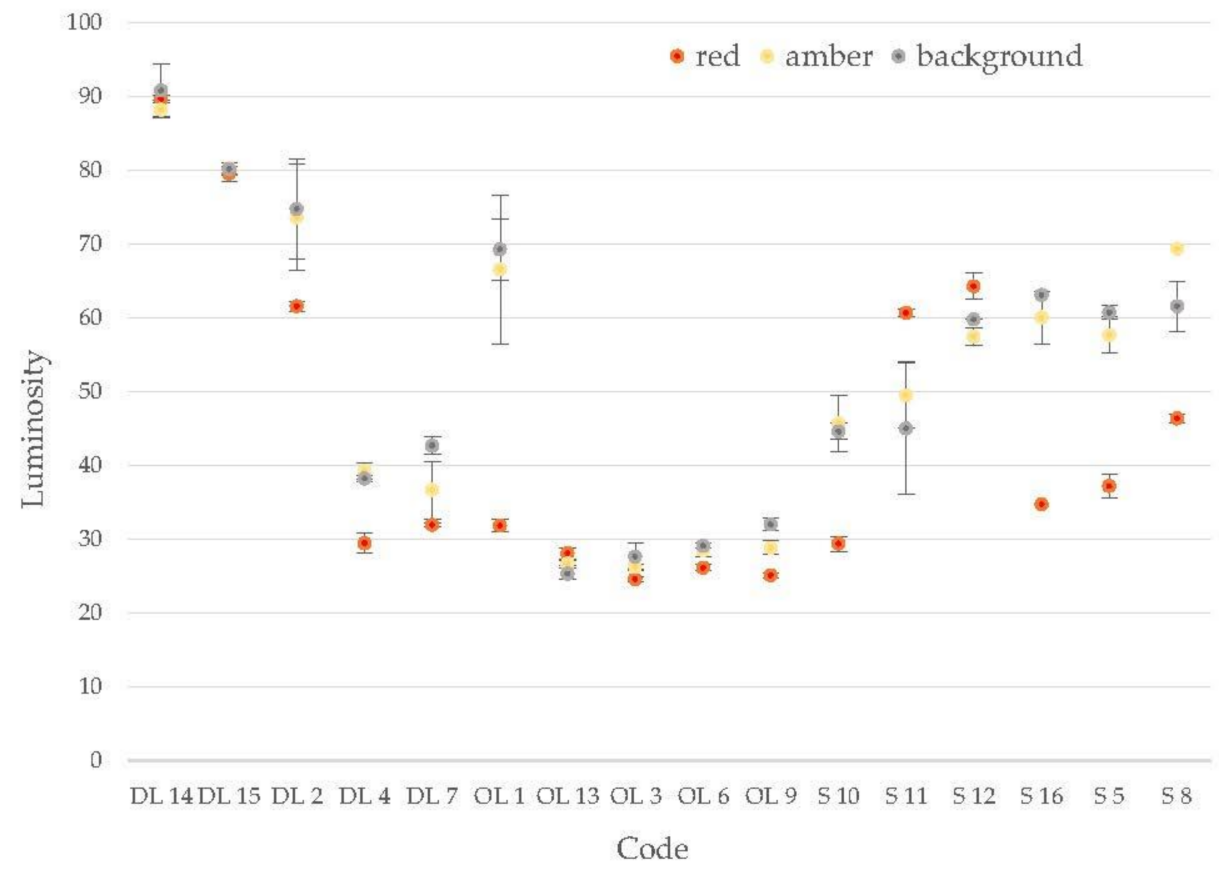

Figure 7. Average of luminosity per color for all the prints. 


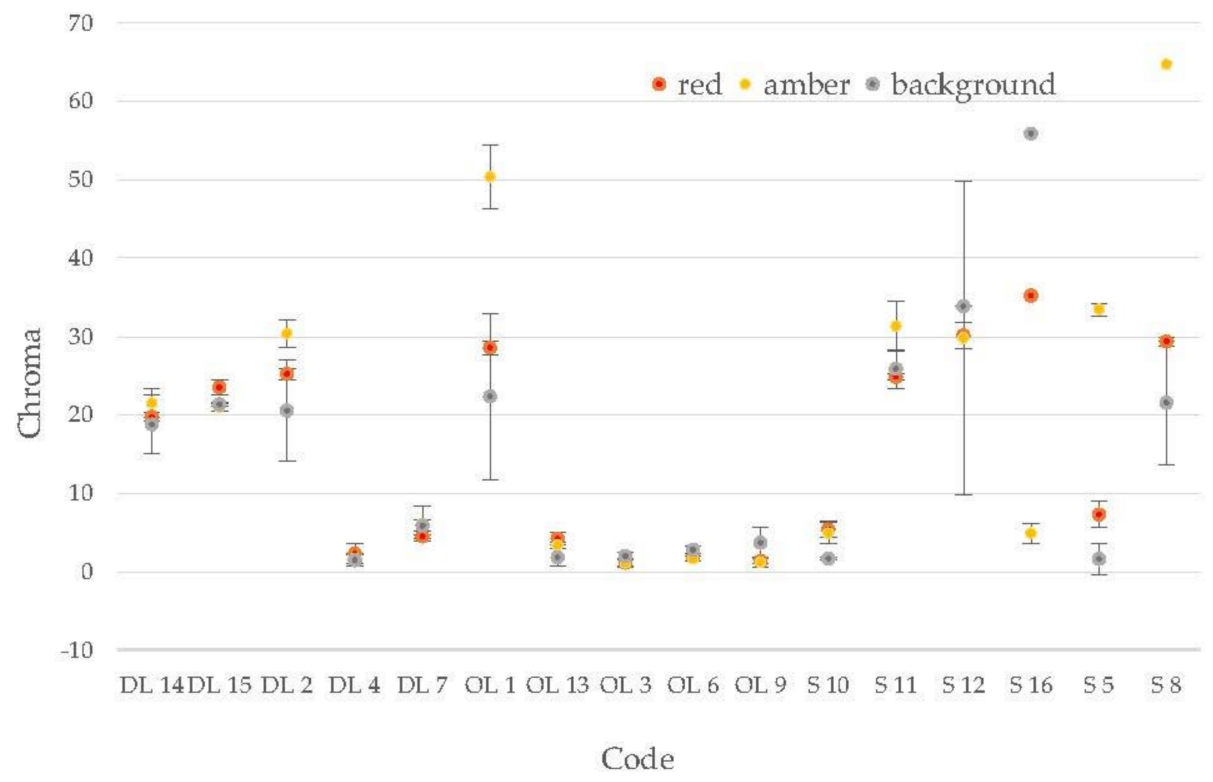

Figure 8. Average of chroma values per color for all the prints.

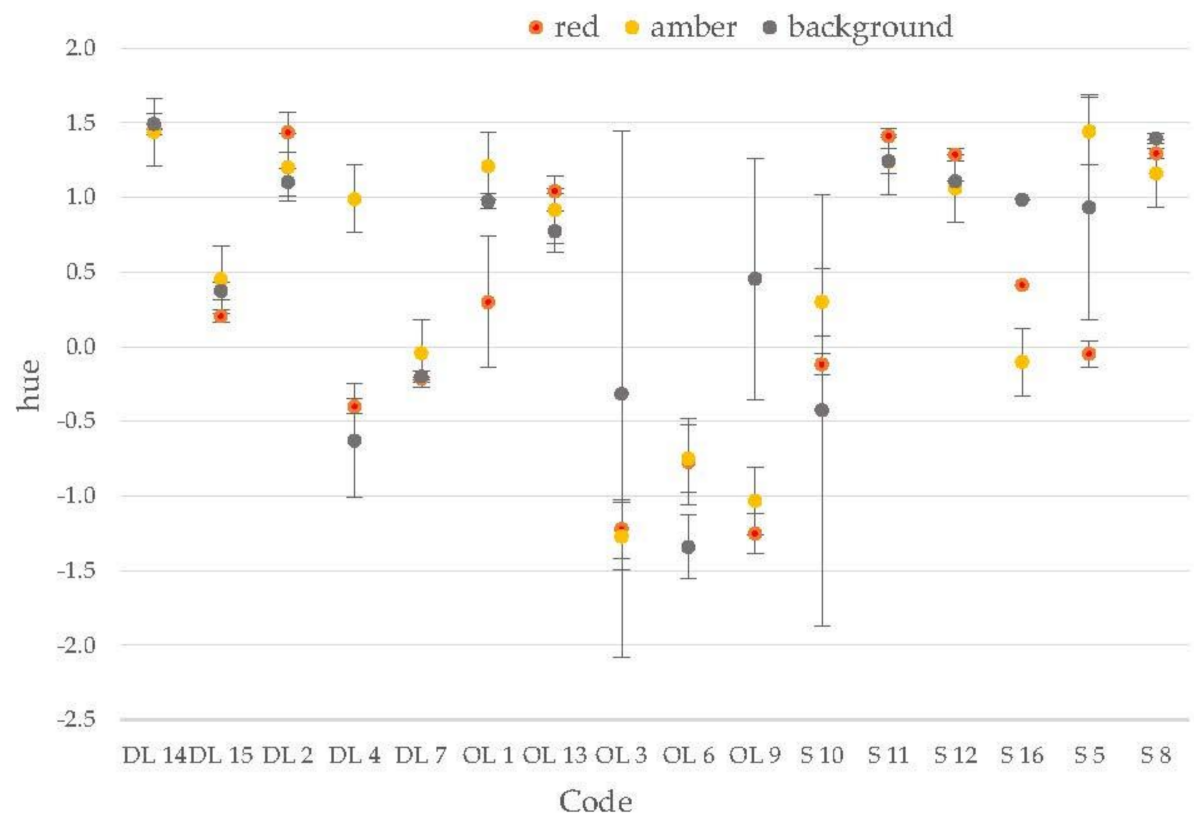

Figure 9. Average of hue values per color for all the prints. 


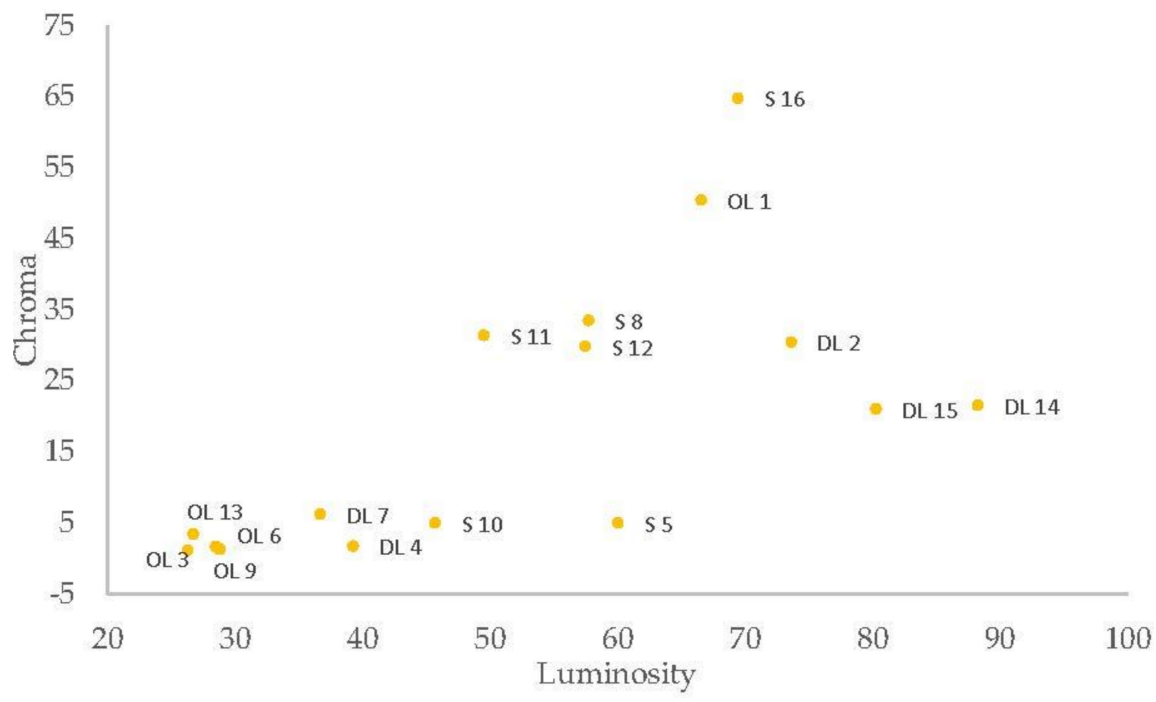

Figure 10. Relation of Chroma (C) vs. Luminosity $\left(\mathrm{L}^{*}\right)$ for average measurements in amber spots.

\section{Discussion}

The images in the Appendix are, except of the CYMK, far from the optical appearance of the actual prints. Structural color, and therefore effect and pearlescent pigments, are notoriously hard to photograph. The wrong lighting and the wrong viewing angle can make an offset lithograph disappear completely. Nevertheless, we will use them to illustrate our findings.

Figures A1, A7-A11 and A13 printed with Spectraval ${ }^{\mathrm{TM}}$ pigments on black paper look pale compared to Figure A3 (OL 1) and Figure A12 (S 16) printed with CMYK inks on white paper. Looking at Figure 4 shows that the $\mathrm{a}^{*}, \mathrm{~b}^{*}$ coordinates of the Spectraval ${ }^{\mathrm{TM}}$ pigments are clustered around the white point, therefore a saturated coloration as in Figures A3 and A12 cannot be achieved. When viewed alone, the brain recalibrates and the changing color as a function of viewing angle overwrites the pale impression with an enhanced 3D perception.

Figures A6, A14, A15 and A17 are much more saturated but very golden, almost mirror like. Even when printed on black paper, Figures A16 and A17 show the overprinting of the different layers, the shadows in the images do not display sufficient grayscale and the image is perceived as metallic but flat. Again, this is not surprising. The $\mathrm{a}^{*}, \mathrm{~b}^{*}$ coordinates of the Iriodin ${ }^{\mathrm{TM}}$ and Pyrisma ${ }^{\mathrm{TM}}$ pigments are all, except Iriodin ${ }^{\mathrm{TM}}$ Icy White, in the upper right quadrant. No blue or green tones can be generated by them.

The printing method has an influence on the image quality. As a consequence of a much thicker ink layer for screen printing, the shadows in the CMYK screen print (Figure A12) shift towards the red compared to the CMYK lithograph (Figure A3), confirmed in Figure 6c, points S16 (screen print) and OL1 (offset lithograph). The measurements in area Amber 2 in Figure 6, points S16 and OL1 in Figure 6a confirm a 'greener' amber in the lithograph. On the other hand, Red 3 in Figure 5 is 'redder' in the lithograph, establishing a bigger gamut for the lithograph. Figure 8 shows that in the lithograph amber and red are more saturated than the background for OL 1. Red in OL 1, however, has less luminosity than the other two colors in consideration (Figure 7).

To combine the best of the worlds, we tried to introduce a color change as function of viewing angle or a metallic sheen by introducing Iriodin ${ }^{\mathrm{TM}}$ Solar Gold in the CMYK print. Iriodin ${ }^{\mathrm{TM}}$ Solar Gold appears as golden yellow when printed on white (Figure A5). Replacing yellow with Iriodin ${ }^{\mathrm{TM}}$ Solar Gold inhibited color mixing. Figure A2 shows that no orange or red tones appeared from the over printing of Iriodin ${ }^{\mathrm{TM}}$ Solar Gold with Primebio $^{\mathrm{TM}}$ Magenta. Solar Gold acts like a mirror. We over-printed yellow with Solar Gold, then magenta, cyan, and finally black to remedy the situation. Figure A4 shows the result. In Figures 6-9 this print is represented by DL2. Even when printed on yellow, Iriodin ${ }^{\mathrm{TM}}$ 
Solar Gold acts like a mirror. Amber and red are desaturated, reduced in chroma and hue. The background starts glowing.

Printing color using inks and dyes are based on the principle of the subtractive color model, which predicts the color of a surface according to the spectral power distribution of light, after it passes through successive layers of partially absorbing media. When printing on white paper, the light passes through microscopic "stacks" of such partially absorbing media and is reflected/scattered back by the printing substrate. Then it passes again through the pigmented medium to be perceived as color by the viewer.

Spectraval $^{\mathrm{TM}}$ inks are perceived as nearly white when observed in powder form; however, they display color when applied to a black substrate. Each ink reflects a portion of the spectrum of the incoming light and transmits the rest. White ink reflects all the spectrum. When applied on white paper, they look white, as the paper will reflect most of the light that is transmitted through the ink. Spectraval ${ }^{\mathrm{TM}}$ pigments do not produce color based on selective absorption as traditional pigments do.Moreover, opposite to the subtractive color model, the output is white instead of black when mixing red, green and blue inks. Previous works have addressed how these pigments allow printing additively [20]. However, the gamut of the Spectraval RGB pigments is much smaller than the gamut of sRGB or Adobe RGB (1998) color spaces, therefore the pigments are considered not suitable for realistic color prints.

In this study, there are several variables to consider: color of the substrate, printing method, concentration of inks, combination of inks and order of deposition, and whether the image was produced from a positive or negative film.

Images were used as positives for subtractive/absorbent inks deposited on white paper, or as negatives for reflective inks applied on black paper. The Amber Orange and Solar Gold pigments are both reflective and absorbing. To understand its behavior, Amber Orange was applied using a positive plate on black paper and as negative on white paper (S11 Figure A15 and OL 13 Figure A6) The prints made on black paper from positives show black areas where there should be white areas depicting the apparent glossiness of the amber beads.

As expected, the variable thickness of the layer affects the saturation, chroma and intensity of the prints. Screen printing produces thicker layers than offset litho or direct litho. Screen printing also contributes to a higher chroma as exampled in S 16 (Figure A12) but not necessarily to a higher luminosity, as it depends on the kind of inks and substrates used.

DL14 (Figure A5) and DL15 (Figure A2) have the highest luminosity. These were images printed on white paper using only Solar Gold ink and Solar Gold plus Magenta ink, respectively. The measurements on amber-colored areas (Figure 7) show that the luminosity is reduced by around $10 \%$ whilst the chroma remains almost the same (Figures 8 and 10). The chroma is increased in the red spots; when the magenta is under-laying the Solar Gold layer the hue is shifted towards the red. These images have a higher luminosity because they were printed on a highly reflective substrate and do not have a dense layer of absorbent pigments

The images printed on white paper with process inks (CMYK) with the methods of direct (DL 2 Figure A4) and offset lithography (OL 1 Figure A3) also have a higher luminosity than the images printed on black, with the exception of S12 (Figure A14). As discussed above, screen printing results in thicker layers. The layer of ink is dense enough to reduce the luminosity, while increasing the chroma of specific colors.

It would be expected that offset litho would give a higher luminosity, as the ink layer would be thinner than using direct litho. DL 2, however, was overprinted with Iriodin ${ }^{\mathrm{TM}}$ Solar Gold resulting in a higher luminosity than using YMCK only. This effect proves the layer acts as a mirror as stated above. When comparing the chromaticity of DL 2 and OL 1, we see that it is considerably higher for OL1, which means that overprinting with Solar Gold, as in DL2, reduces the intensity of the color of amber and red beads. Observing the measurements of background — which are not altered by overprinting Solar Gold-we 
notice that the luminosity is not considerably affected, whilst the chroma of DL 2 is higher (as the pigment layer is thicker).

Among the prints on black paper using Spectraval ${ }^{\mathrm{TM}}$ inks, screen printing gives higher luminosity values than lithography as the ink layer is thicker (S5 Figure A1, S8 Figure A13, S10). There is a difference in the concentration of pigments used between these images; Figure A13 was made with half the amount of pigment compared to Figure A1, therefore its reflectivity is lower.

The RGB print made by direct lithography (DL7 Figure A8 has a higher luminosity than the ones made by offset litho (OL 9 Figure A7, OL 6 Figure A10 and OL 3) also due to the layer thickness. The difference between OL 9, OL 6, OL3 is the number of passes. They have two, three and four passes of blue ink, respectively. Increasing the passes of the blue layer, reduced the luminosity of the prints although the hue is not changed in an evident way (Figure 9).

By visual inspection and from its color coordinates we see that DL 7 has a reddish appearance, which did not happen to Figure A9, printed by the same method. The explanation for this is the fact that the red layer was printedlast.

When using Iriodin ${ }^{\mathrm{TM}}$ and Prysma ${ }^{\mathrm{TM}}$ inks, screen printing on white produced the highest luminosity (S12 Figure A14) (White Positive I \& P, dark to light), followed by screening print with I \& P inks on black paper (S11 Figure A15, dark to light)—where the inks covers well the black substrate. The image made with Solar gold on Y plate over black paper by direct litho (DL 4 Figure A9) produced less luminous prints than screen printing. The positive image made by offset litho (OL 13 Figure A6) as a positive on black with I \& P inks (light to dark) had the lowest luminosity among the set.

Although it was not measured, visually, Figure A17, produced by screen printing using negatives, has a higher chromaticity than Figure A16 made by lithography also from a negative on black paper. Finally, it was observed that screen printing generally increases the contrast of the images.

\section{Conclusions}

Spectraval ${ }^{\mathrm{TM}}$ pigments produce inks with a limited chroma compared to CMYK inks, therefore producing equally saturated prints is impossible. The brain, nevertheless, is able to interpret the pale impression of prints produced with Spectraval ${ }^{\mathrm{TM}}$ based inks and its angle-dependent color, as an enhanced 3D perception. Iriodin ${ }^{\mathrm{TM}}$ and Pyrisma ${ }^{\mathrm{TM}}$ produce more saturated prints, but with an overall golden appearance, which aligns with their color coordinates. No blue or green tones can be generated by them. When comparing lithographic and screen-printing prints for CMYK inks, it was observed that a much thicker ink layer has a smaller gamut.

Creating a 3D color experience is more complex than expected. Printing with RGB Spectraval ${ }^{\mathrm{TM}}$ pigments alone mimics the 'traveling light' in the image and creates a 3D effect, but color saturation is low. Introducing an effect layer into a traditional CMYK print shifts the color of the print in an unexpected way. These experiments are not conclusive on what is the best way to print amber. However, practical knowledge, as well as visual and quantitative results, has been gained. Such outputs are relevant to the use of reflective inks in the lithographic and screen-printing process, from both the artistic and the scientific perspective. Future work will include the development of a pipeline for color management. Therefore, prior understanding of the methods and their limitations is essential in the design of the subsequent experiments, such as printing color charts and developing a perceptual color model. Moreover, in the future, we will explore how we can exploit the combination of absorbing pigments and structural color, known from nature, to generate a full-color, pseudo-3D print. 
Author Contributions: Conceptualization, C.P. and S.K.; methodology S.K. and C.P.; validation, A.T.-V., H.F. and S.K.; formal analysis, A.T.-V.; investigation, S.K., H.F., A.T.-V.; resources, C.P. and S.K.; writing-original draft preparation, S.K., A.T.-V., H.F.; writing-review and editing, S.K.; visualization, A.T.-V.; supervision, C.P. and S.K.; project administration, C.P.; funding acquisition, C.P. All authors have read and agreed to the published version of the manuscript.

Funding: This research was funded with the support of EPSRC grant EP/R011761/1, ApPEARS Appearance Printing European Advanced Printing School European Union's Horizon 2020 research and innovation programme under the MSCA ITN-ETN grant agreement No. 814158 and Research England Expanding Excellence in England Fund 2019/22: Centre for Print Research. The project was supported by Merck, by kindly donating the pigments. We would like to thank William Andre for helping with the printing.

Institutional Review Board Statement: Not applicable.

Data Availability Statement: The data presented in this study are openly available in UWE data repository [online]. Available online: http:/ / researchdata.uwe.ac.uk/646 (accessed on 30 October 2021).

Conflicts of Interest: The authors declare no conflict of interest.

\section{Appendix A}

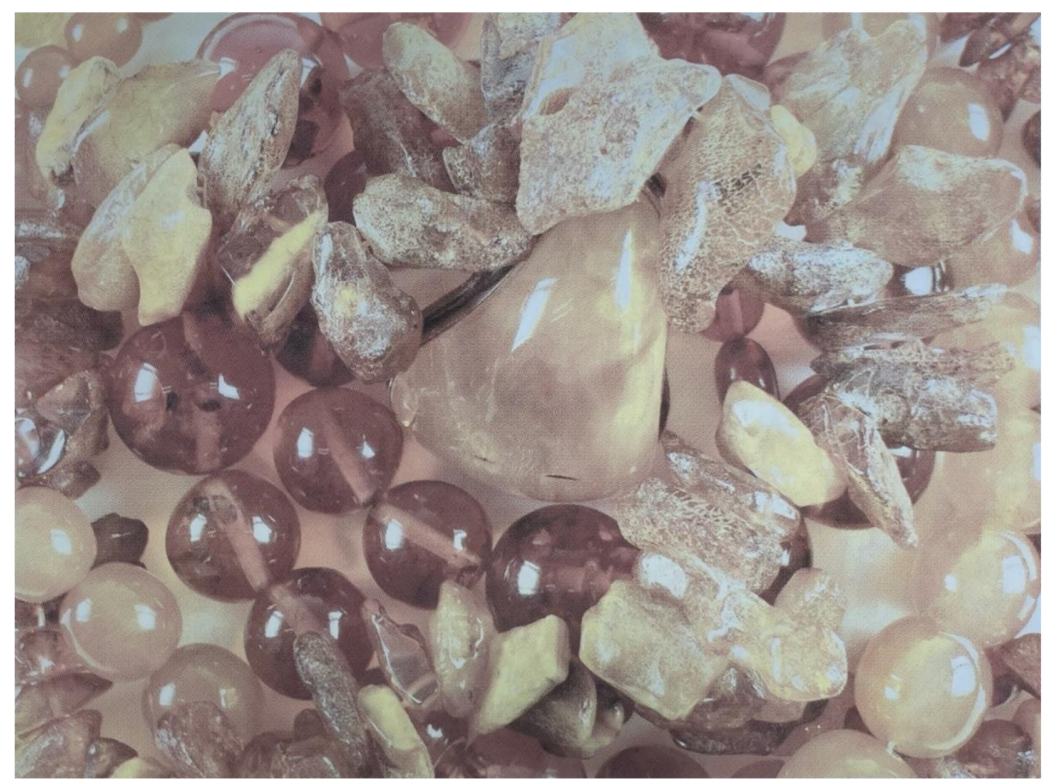

Figure A1. 25LPI, screen-print, 55H mesh, Spectraval ${ }^{\mathrm{TM}}$ pigments, RGB, $\mathrm{R}=400 \mathrm{~g}$ medium, 36 g pigment, $\mathrm{G}=400 \mathrm{~g}$ medium, $48 \mathrm{~g}$ pigment, $\mathrm{B}=400 \mathrm{~g}$ medium, $40 \mathrm{~g}$ pigment, black Plike, film negative, print size $600 \mathrm{~mm} \times 450 \mathrm{~mm}$. 


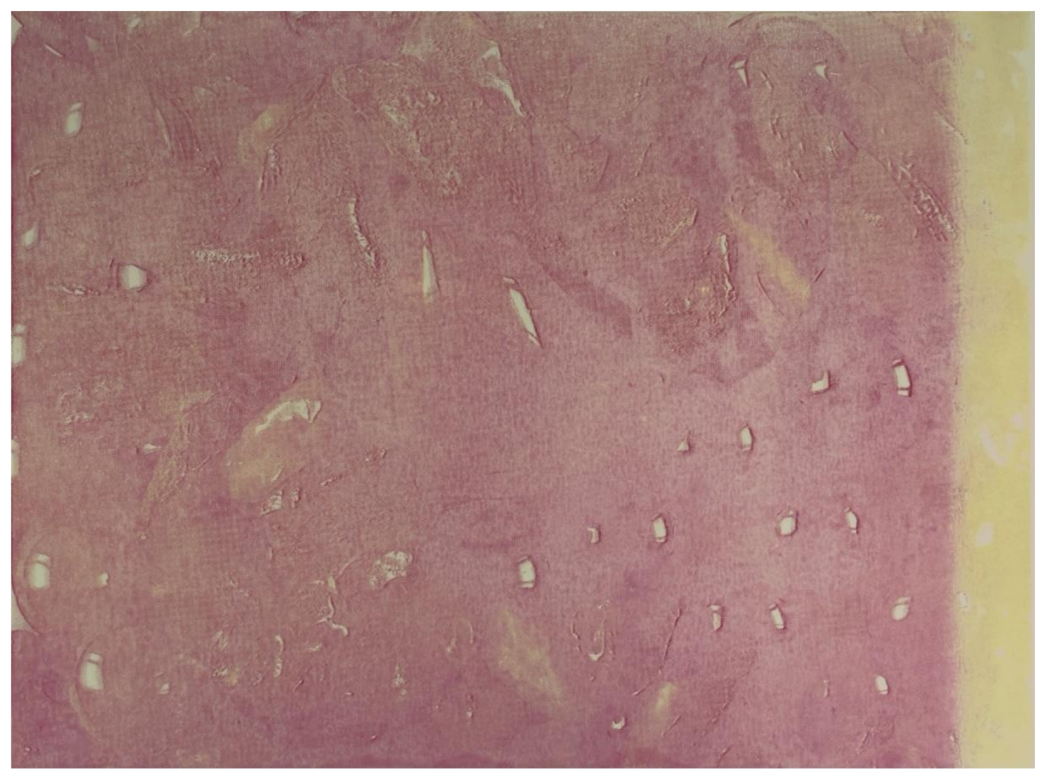

Figure A2. 25LPI, direct litho, Iriodin ${ }^{\mathrm{TM}}$ Solar Gold on yellow plate overprinted with magenta, white Plike, film positive, print size: $600 \mathrm{~mm} \times 450 \mathrm{~mm}$.

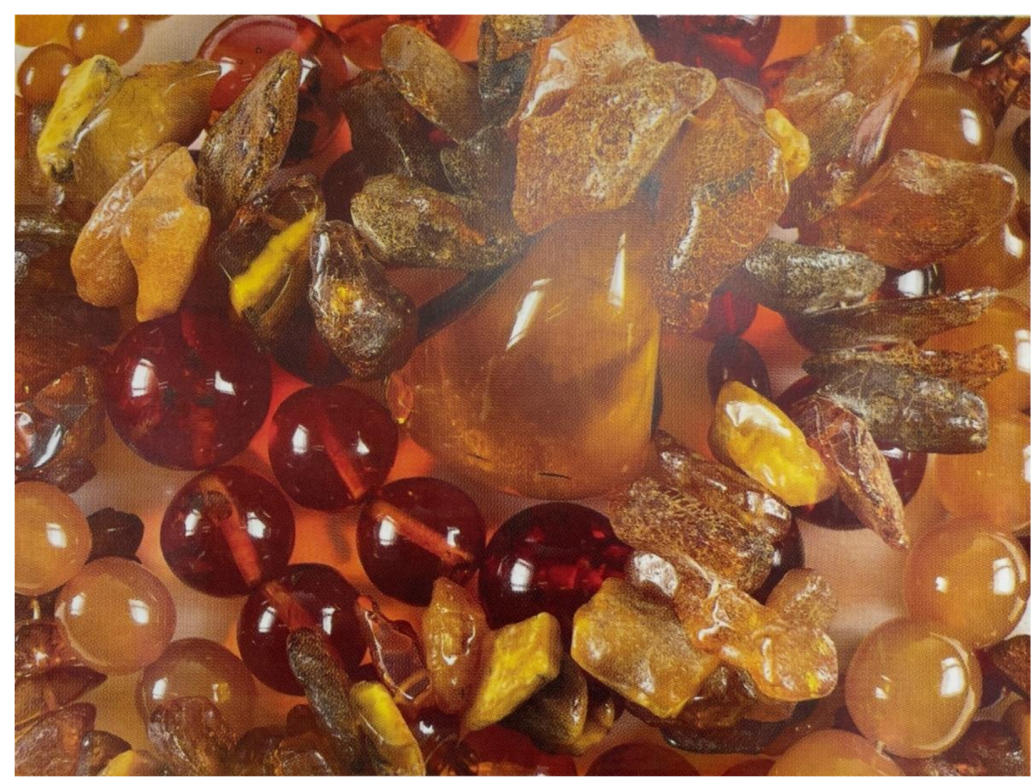

Figure A3. 25LPI, offset litho, CMYK inks, printed $\mathrm{Y}, \mathrm{M}, \mathrm{C}, \mathrm{K}$, white Plike, film positive, print size: $600 \mathrm{~mm} \times 450 \mathrm{~mm}$. 


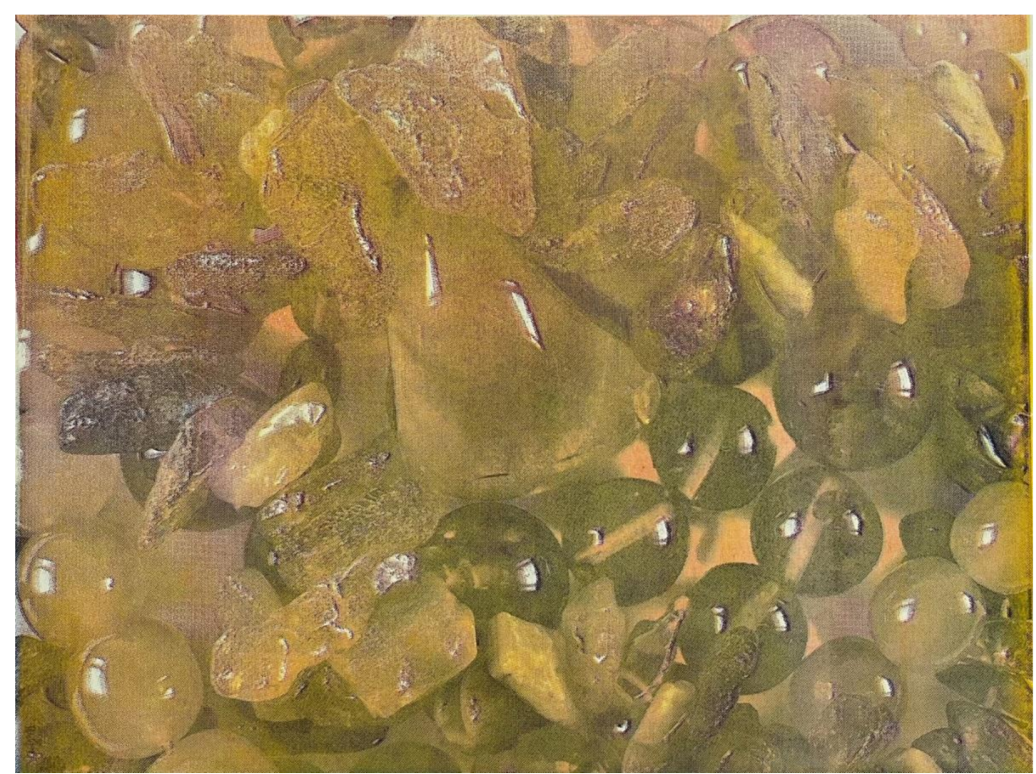

Figure A4. 25LPI, direct litho, YMCK, Y overprinted with Iriodin ${ }^{\mathrm{TM}}$ Solar Gold, white Plike, film positive, print size: $600 \mathrm{~mm} \times 450 \mathrm{~mm}$.

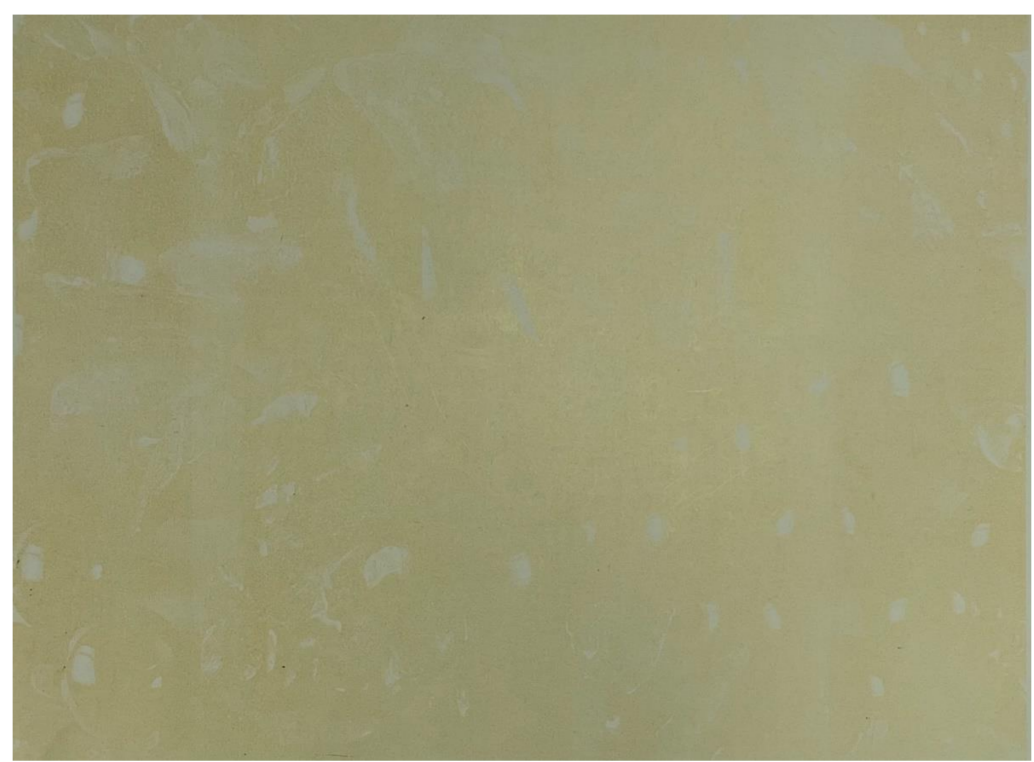

Figure A5. 25LPI, direct litho, Iriodin ${ }^{\mathrm{TM}}$ Solar Gold on yellow plate, white Plike, film positive, print size: $600 \mathrm{~mm} \times 450 \mathrm{~mm}$. 


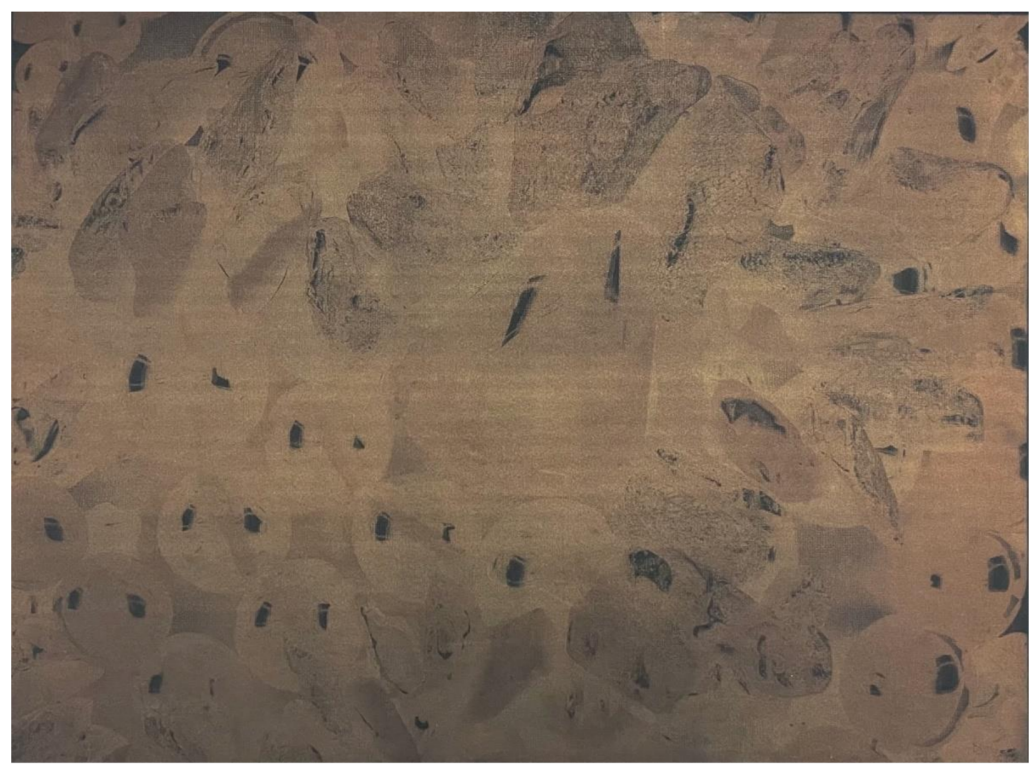

Figure A6. 25LPI, offset litho, $\mathrm{C}=$ Iriodin $^{\mathrm{TM}}$ Icy White, $\mathrm{B}=$ Iriodin $^{\mathrm{TM}}$ Platinum Gold, $\mathrm{M}=$ Iriodin $^{\mathrm{TM}}$ Solar Gold, $\mathrm{Y}=$ Pyrisma $^{\mathrm{TM}}$ Ambercup Orange, printed light to dark, black Plike, film positive, print size: $600 \mathrm{~mm} \times 450 \mathrm{~mm}$.

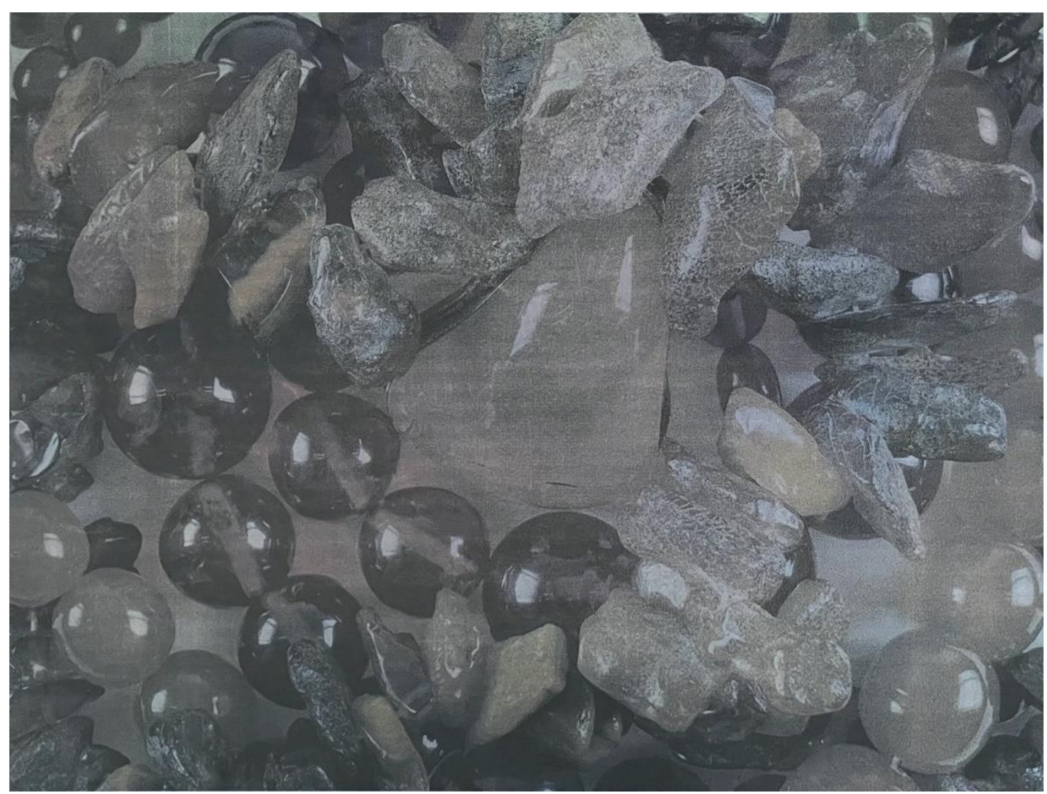

Figure A7. 25LPI, offset litho, Spectraval ${ }^{\mathrm{TM}}$ pigments, $\mathrm{R}=2 \times$ pass, $\mathrm{G}=3 \times$ pass, $\mathrm{B}=2 \times$ pass, black Plike, film negative, print size: $600 \mathrm{~mm} \times 450 \mathrm{~mm}$. 


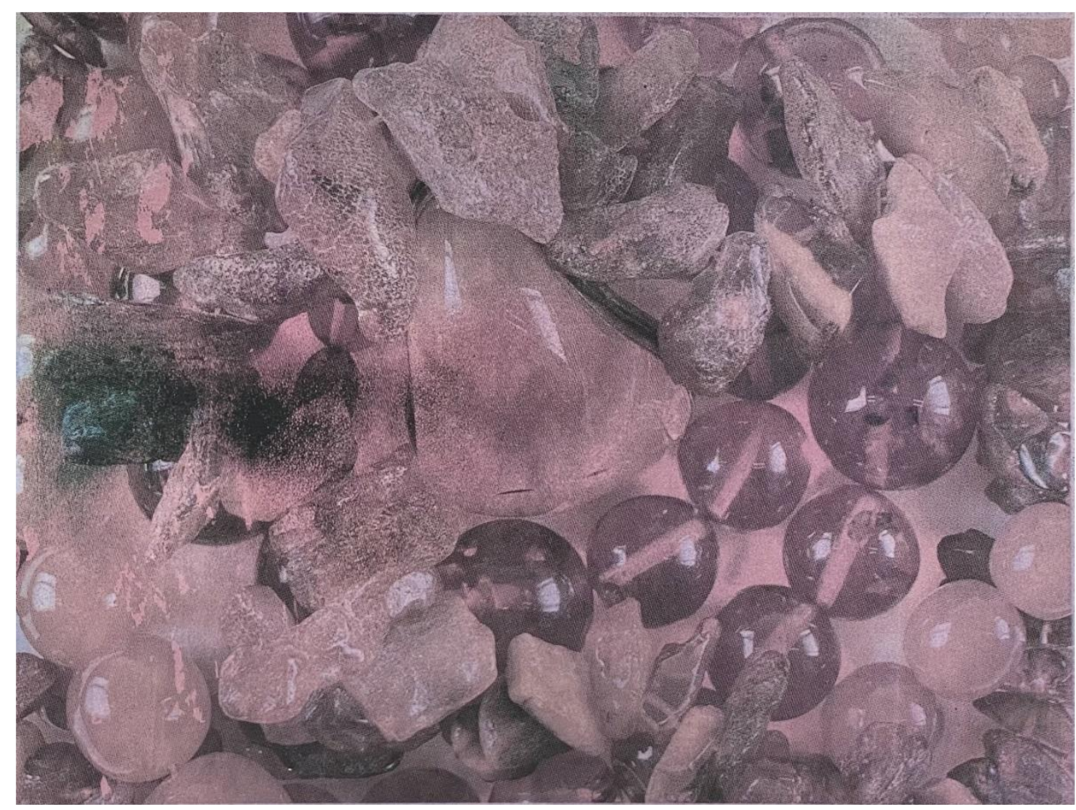

Figure A8. 25LPI, direct litho, Spectraval ${ }^{\mathrm{TM}}$ pigments, B,G,R, black Plike, film negative, print size: $600 \mathrm{~mm} \times 450 \mathrm{~mm}$.

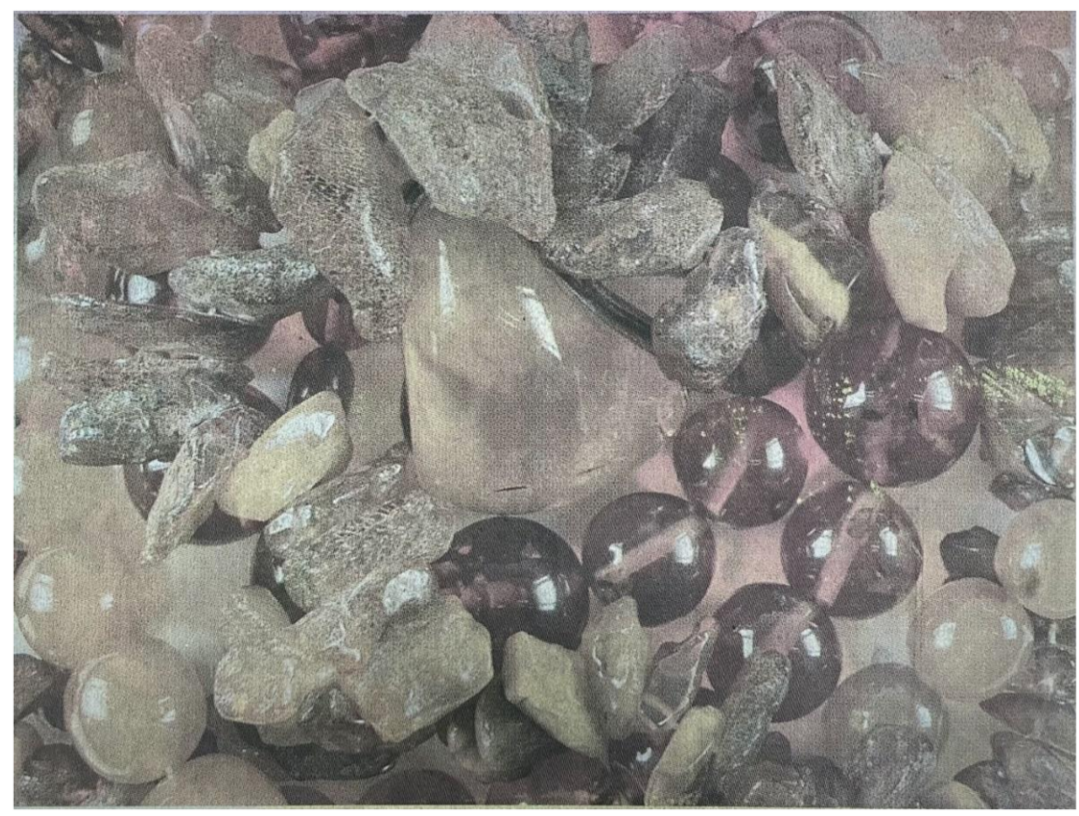

Figure A9. 25LPI, direct litho, Spectraval ${ }^{\mathrm{TM}}$ pigments, $R, G, B$, black Plike, film negative, print size: $600 \mathrm{~mm} \times 450 \mathrm{~mm}$. 


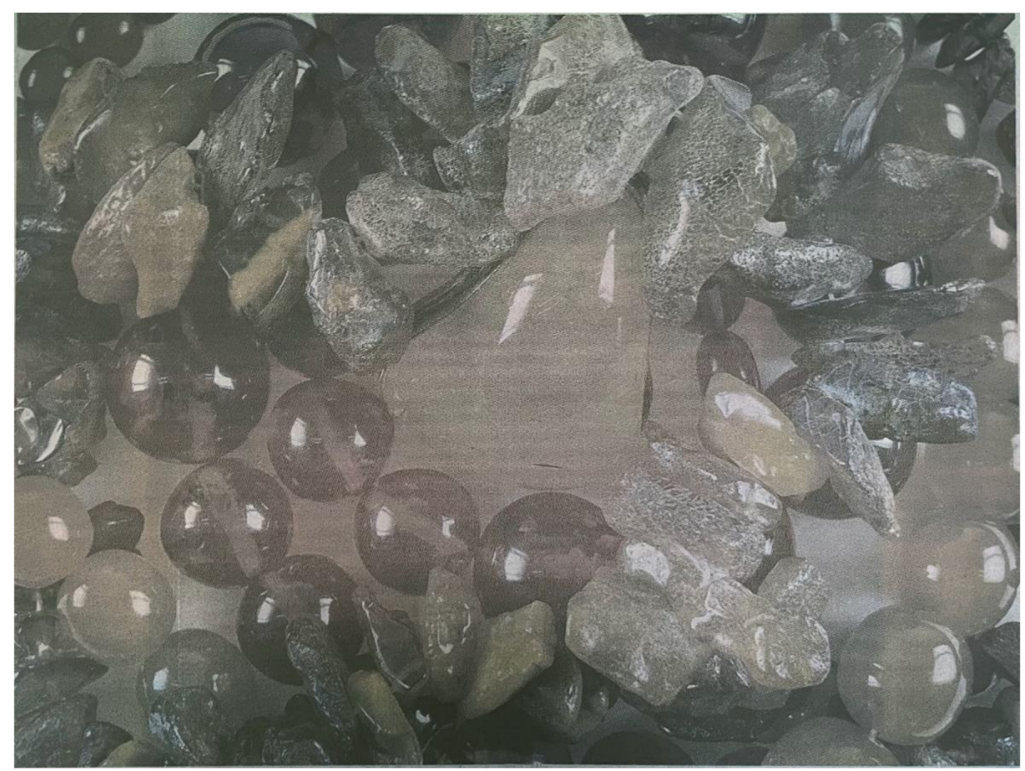

Figure A10. 25LPI, offset litho, Spectraval ${ }^{\mathrm{TM}}$ pigments, $\mathrm{R}=2 \times$ pass, $\mathrm{G}=3 \times$ pass, $\mathrm{B}=3 \times$ pass, black Plike, film negative, print size: $600 \mathrm{~mm} \times 450 \mathrm{~mm}$.

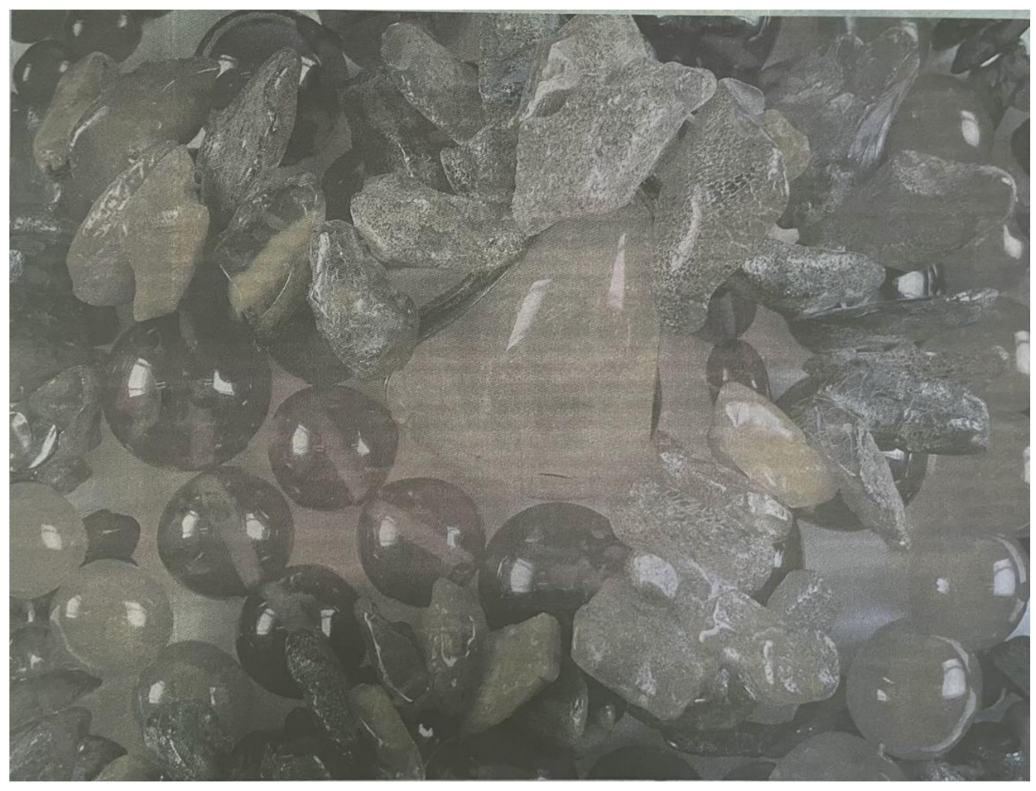

Figure A11. 25LPI, offset litho, Spectraval ${ }^{\mathrm{TM}}$ pigments, $\mathrm{R}=2 \times$ pass, $\mathrm{G}=3 \times$ pass, $\mathrm{B}=4 \times$ pass black Plike, film negative, print size: $600 \mathrm{~mm} \times 450 \mathrm{~mm}$. 


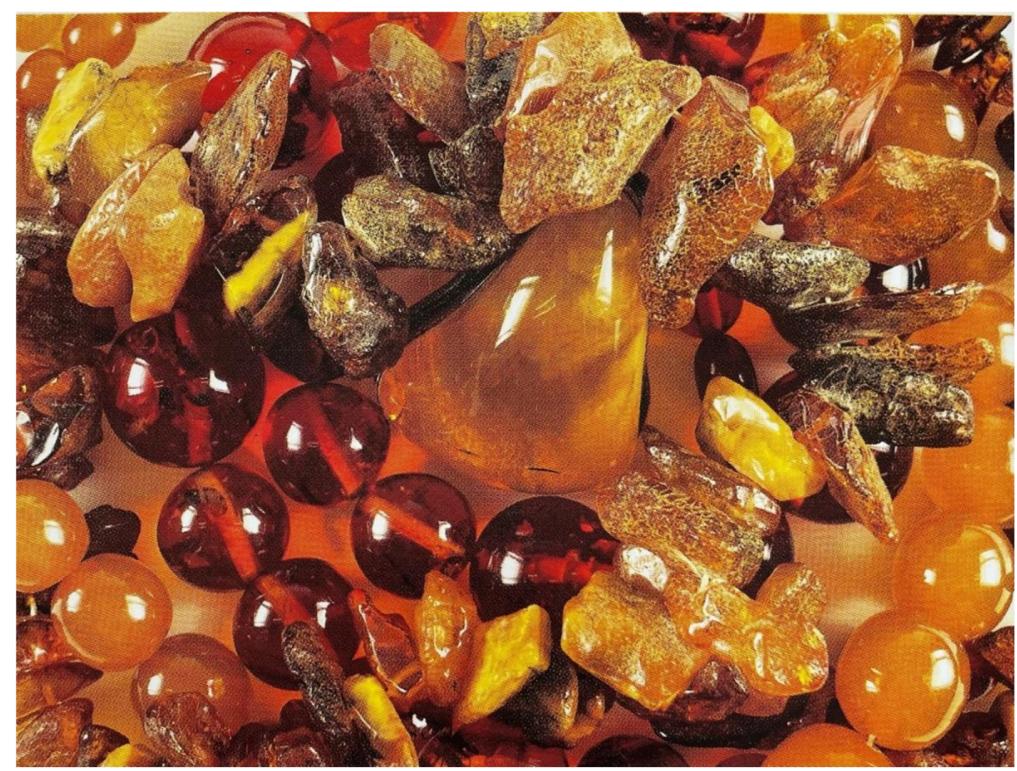

Figure A12. 25LPI, screen-print, 55H mesh, CMYK, printed $\mathrm{Y}, \mathrm{M}, \mathrm{C}$, K, white Plike, film positive, print size: $600 \mathrm{~mm} \times 450 \mathrm{~mm}$.

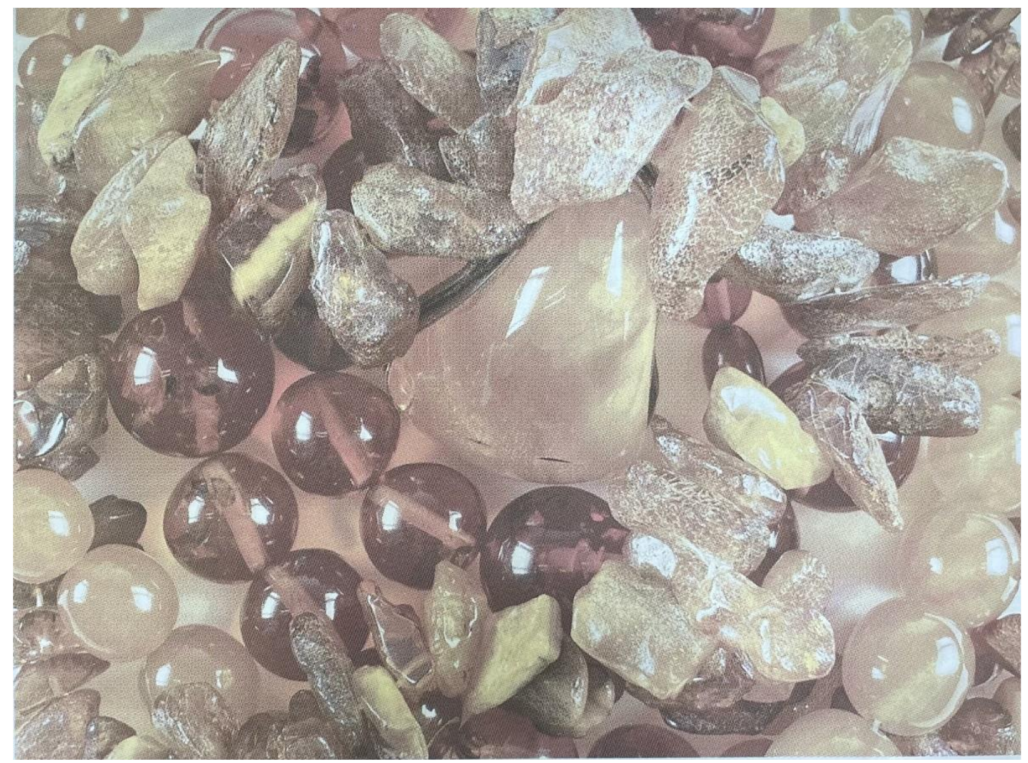

Figure A13. 25LPI screen-print, 55H mesh, Spectraval ${ }^{\mathrm{TM}}$ pigments, $\mathrm{R}=400 \mathrm{~g}$ medium, $18 \mathrm{~g}$ pigment, $\mathrm{G}=400 \mathrm{~g}$ medium, $24 \mathrm{~g}$ pigment, $\mathrm{B}=400 \mathrm{~g}$ medium, $20 \mathrm{~g}$ pigment, black Plike, film negative, print size: $600 \mathrm{~mm} \times 450 \mathrm{~mm}$. 


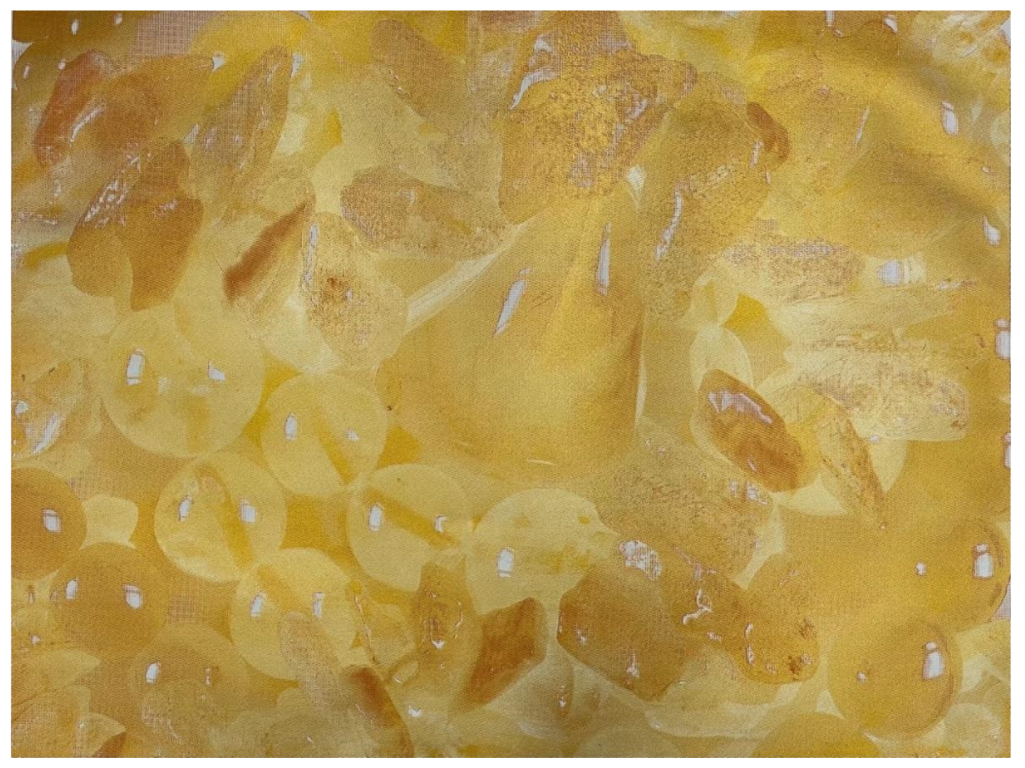

Figure A14. 25LPI, screen-print, 55H mesh, $\mathrm{Y}=$ Pyrisma $^{\mathrm{TM}}$ Ambercup Orange, $\mathrm{M}=$ Iriodin $^{\mathrm{TM}}$ Solar Gold, B = Iriodin ${ }^{\mathrm{TM}}$ Platinum Gold, C = Iriodin ${ }^{\mathrm{TM}}$ Icy White, printed dark to light, white Plike, film positive, print size: $600 \mathrm{~mm} \times 450 \mathrm{~mm}$.

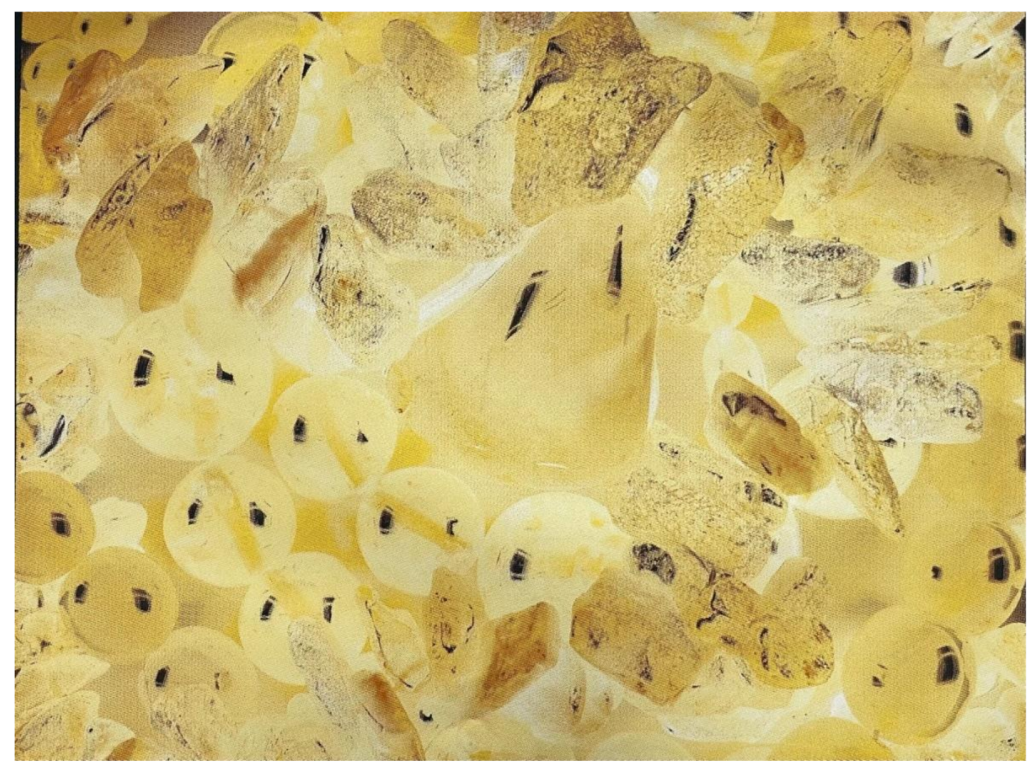

Figure A15. 25LPI, screen-print, 55H mesh, $\mathrm{Y}=$ Pyrisma $^{\mathrm{TM}}$ Ambercup Orange, $\mathrm{M}=$ Iriodin $^{\mathrm{TM}}$ Solar Gold, B = Iriodin ${ }^{\mathrm{TM}}$ Platinum Gold, C = Iriodin ${ }^{\mathrm{TM}}$ Icy White, printed dark to light, black Plike, film positive, print size: $600 \mathrm{~mm} \times 450 \mathrm{~mm}$. 


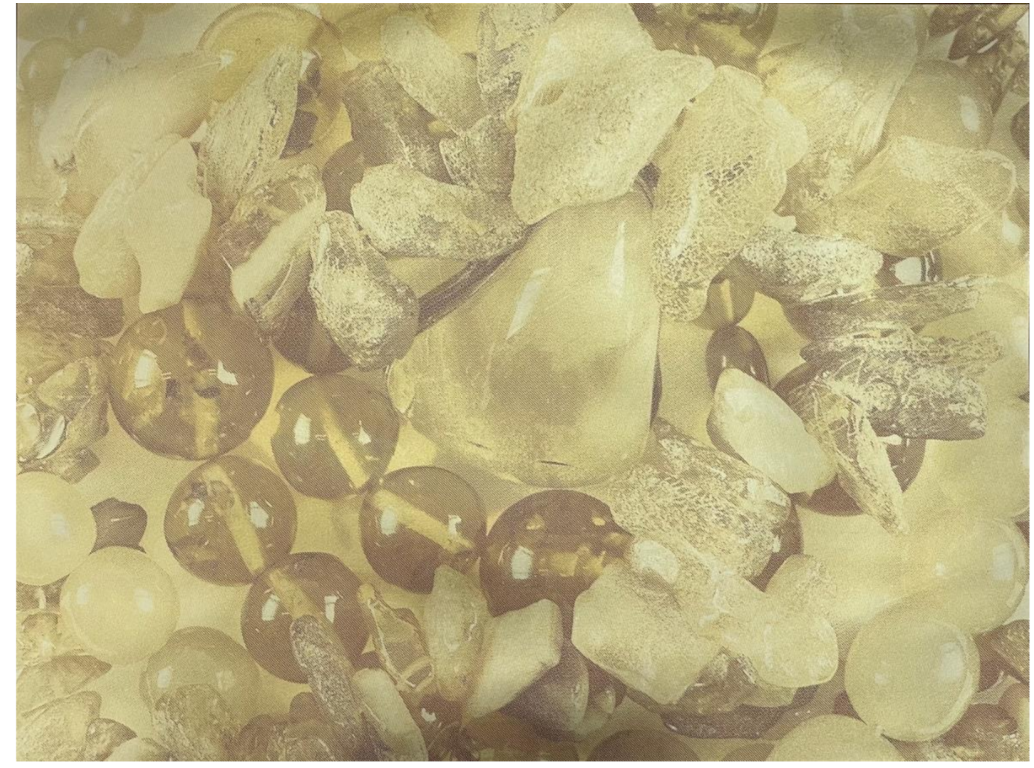

Figure A16. 25LPI, C = Pyrisma ${ }^{\mathrm{TM}}$ Ambercup Orange, $\mathrm{B}=\operatorname{Iriodin}^{\mathrm{TM}}$ Solar Gold, $\mathrm{M}=\operatorname{Iriodin}^{\mathrm{TM}}$ Platinum Gold, printed dark to light, black Plike, film negative, print size: $600 \mathrm{~mm} \times 450 \mathrm{~mm}$.

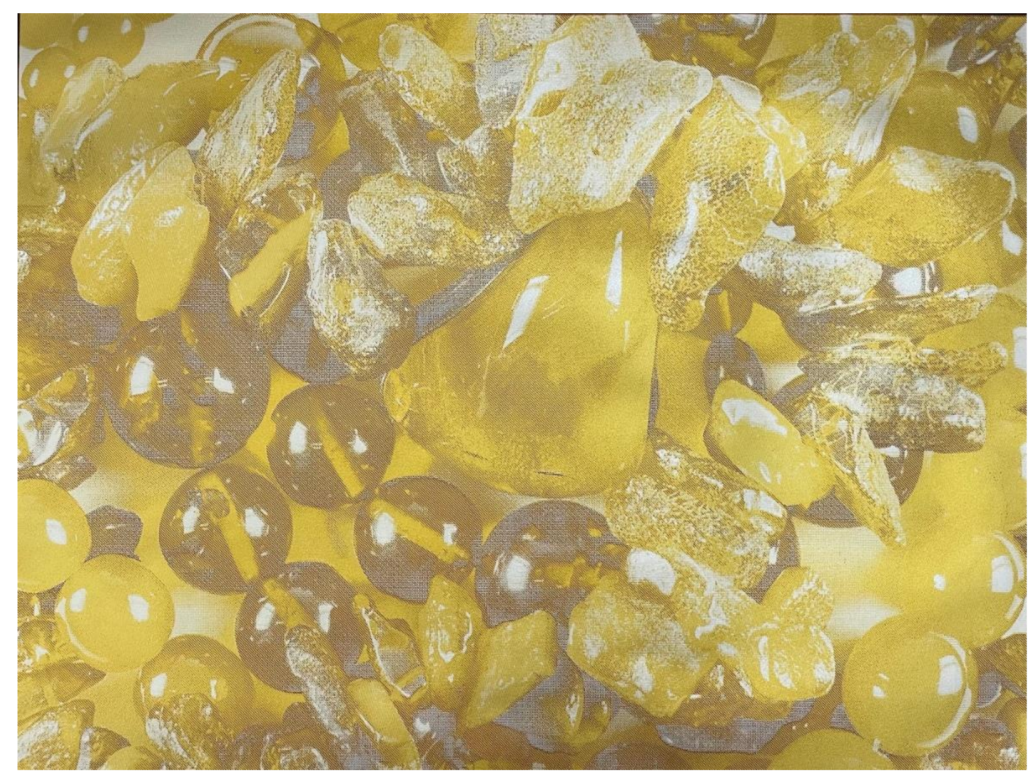

Figure A17. 25LPI, $C=$ Pyrisma $^{\mathrm{TM}}$ Ambercup Orange, $\mathrm{B}=$ Iriodin $^{\mathrm{TM}}$ Solar Gold, $\mathrm{M}=\operatorname{Iriodin}^{\mathrm{TM}}$ Platinum Gold, $\mathrm{Y}=$ Iriodin $^{\mathrm{TM}}$ Icy White, printed dark to light, black Plike, film negative, print size: $600 \mathrm{~mm} \times 450 \mathrm{~mm}$.

\section{References}

1. Falk, D.; Brill, D. David Stork: Seeing the Light. Optics in Nature, Photography, Color, Vision, and Holography; Echo Point Books \& Media: Brattleboro, VT, USA, 2020.

2. Loske, A.; Colour, A. Visual History; ILEX: Lewes, UK, 2019.

3. Eckstut, J.; Eckstut, A. The Secret Language of Color; Black Dog \& Leventhal Publishers: New York, NY, USA, 2013.

4. Parraman, C.; Klein, S. Printing the light. Color. Technol. 2021, 137, 86-89. [CrossRef]

5. Carter, K. How to Print Successfully Using Soft and Hard Proofs. Canon Europe. 2021. Available online: https://www.canoneurope.com/pro/stories/soft-hard-proofing/ (accessed on 31 October 2021).

6. Maile, F.J.; Pfaff, G.; Reynders, P. Effect pigments-Past, present and future. Prog. Org. Coat. 2005, 54, 150-163. [CrossRef]

7. Merck. Effect Pigments. 2021. Available online: https://www.merckgroup.com/en/expertise/effect-pigments/services/basicpigment-technology.html (accessed on 31 October 2021). 
8. Merck. RGB Printing. 2021. Available online: https://www.merckgroup.com/en/expertise/effect-pigments/solutions/printing/ rgb.html (accessed on 31 October 2021).

9. Kipphan, H. Printing Technologies with Permanent Printing Master. In Handbook of Print Media; Kipphan, H., Ed.; Springer: Berlin/Heidelberg, Germany, 2001. [CrossRef]

10. Association FoFT. FLEXOGRAPHY: Principles \& Practices 6.0; Association FoFT: Bohemia, NY, USA, 2013.

11. Print Club London. Screen Printing. The Ultimate Studio Guide, from Sketchbook to Squeegee; Thames and Hudson: London, UK, 2017; pp. 18-19.

12. Spruill, S.J. Analysis of Ink Film Thickness on Screenless Lithographic Plates by Microscopy; School of Printing Rochester Institute of Technology, 1982; Available online: https:/ / scholarworks.rit.edu/theses/5048/ (accessed on 30 October 2021).

13. Horwood, R. Towards a Better Understanding of Screen Print Thickness Control. Electrocompon. Sci. Technol. 1974, 1, 129-136. [CrossRef]

14. Kawauchi, I.; Nagashima, A.; Mizutani, K. Positive-Working Presensitized Plate for Use in Making Lithographic Printing Plate. European Patent 0589309A1, 10 September 1993.

15. Merck. Effect Pigments: Color Card Print; KGaA M: Darmstadt, Germany, 2021.

16. Atlas Screen Supply Company. Available online: https://www.atlasscreensupply.com/blog/choosing-the-right-mesh-andhalftone-dot.htm (accessed on 30 October 2021).

17. Yanoff, M.; Duker, J.S. Ophthalmology, 5th ed.; Elsevier: Amsterdam, The Netherlands, 2019; Volume xxiii, p. 1411.

18. Heijl, A.; Lindgren, G.; Olsson, J. Normal Variability of Static Perimetric Threshold Values Across the Central Visual Field. Arch. Ophthalmol. 1987, 105, 1544-1549. [CrossRef] [PubMed]

19. Ly, B.C.K.; Dyer, E.B.; Feig, J.L.; Chien, A.L.; Del Bino, S. Research Techniques Made Simple: Cutaneous Colorimetry: A Reliable Technique for Objective Skin Color Measurement. J. Investig. Dermatol. 2020, 140, 3-12.e1. [CrossRef] [PubMed]

20. Klein, S.; Parraman, C.; Voges, L. How to print a rainbow. NIP Digit. Fabr. Conf. 2019, 2019, 52-55. [CrossRef] 